\title{
ATF-4 and hydrogen sulfide signalling mediate longevity from inhibition of translation or mTORC1
}

Cyril Statzer ${ }^{1 \S}$, Jin Meng ${ }^{2,3 \S}$, Richard Venz ${ }^{1}$, Monet Bland ${ }^{2,3}$, Stacey Robida-Stubbs ${ }^{2,3}$, Krina

Patel $^{2,3}$, Dunja Petrovic ${ }^{5}$, Raffaella Emsley ${ }^{4}$, Pengpeng Liu ${ }^{6}$, lanessa Morantte ${ }^{7}$, Cole Haynes $^{6}$, William B. Mair ${ }^{7}$, Alban Longchamp ${ }^{4}$, Milos Filipovic ${ }^{5}$, T. Keith Blackwell ${ }^{2,3^{*}}$, and Collin Y. Ewald ${ }^{1 *}$

1 Eidgenössische Technische Hochschule Zürich, Department of Health Sciences and Technology, Institute of Translational Medicine, Schwerzenbach, Switzerland

2 Department of Genetics, Harvard Medical School, Boston, MA, United States

3 Joslin Diabetes Center, Research Division, Boston, MA, United States

4 Department of Vascular Surgery, Centre Hospitalier Universitaire Vaudois and University of Lausanne, Lausanne, Switzerland

5 Leibniz-Institut für Analytische Wissenschaften-ISAS-e.V., Dortmund, Germany

6 Department of Molecular, Cell and Cancer Biology, University of Massachusetts Medical School, Worcester, MA, U.S.A.

7 Department of Genetics and Complex Diseases, Harvard School of Public Health, 665 Huntington Avenue, Boston, MA, U.S.A.

$\S$ Authors contributed equally

*Corresponding authors: collin-ewald@ethz.ch (C.Y.E.) and keith.blackwell@joslin.harvard.edu (T.K.B.). These authors jointly supervised the work.

Keywords: mRNA translation, cystathionine gamma-lyase, $\mathrm{H}_{2} \mathrm{~S}$, ageing, mTORC1, integrated stress response, $C$. elegans 


\section{Abstract}

Inhibition of the master growth regulator mTORC1 (mechanistic target of

3 rapamycin complex 1) slows ageing across phyla, in part by reducing protein

4 synthesis. Various stresses globally suppress protein synthesis through the integrated

5 stress response (ISR), resulting in preferential translation of the transcription factor

6 ATF-4. Here we show in C. elegans that inhibition of translation or mTORC1 increases

7 ATF-4 expression, and that ATF-4 mediates longevity under these conditions

8 independently of ISR signalling. ATF-4 promotes longevity by activating canonical

9 anti-ageing mechanisms, but also by elevating expression of the transsulfuration

10 enzyme $\mathrm{CTH}-2$ to increase hydrogen sulfide $\left(\mathrm{H}_{2} \mathrm{~S}\right)$ production. This $\mathrm{H}_{2} \mathrm{~S}$ boost

11 increases protein persulfidation, a protective modification of redox-reactive cysteines.

12 The ATF-4/CTH-2/ $\mathrm{H}_{2} \mathrm{~S}$ pathway also mediates longevity and increased stress

13 resistance from mTORC1 suppression. Increasing $\mathrm{H}_{2} \mathrm{~S}$ levels, or enhancing

14 mechanisms that $\mathrm{H}_{2} \mathrm{~S}$ influences through persulfidation, may represent promising

15 strategies for mobilising therapeutic benefits of the ISR, translation suppression, or

16 mTORC1 inhibition. 


\section{Introduction}

Over the last three decades, genetic and phenotypic analyses of ageing have revealed that across eukaryotes, lifespan can be extended by inhibition of mechanisms that promote growth and proliferation ${ }^{1-3}$. Prominent among these is the serine/threonine kinase complex mTORC1, which coordinates the activity of multiple growth-related processes in response to growth factor and nutrient signals ${ }^{2-4}$. mTORC1 activity can be reduced by genetic perturbations, dietary restriction (DR), or pharmacological interventions such as rapamycin, an mTORC1 inhibitor that increases lifespan from yeast to mice ${ }^{2,3}$. However, while rapamycin represents a very exciting paradigm for anti-ageing pharmacology, mTORC1 suppression has wideranging effects on the organism ${ }^{2,3}$. Rapamycin is used clinically as an immunosuppressant, and mTORC1 broadly affects metabolism and supports the synthesis of proteins, nucleic acids, and lipids ${ }^{2-4}$. Elucidation of specific mechanisms through which mTORC1 influences longevity is critical not only for understanding the biology of ageing and longevity, but also for the development of molecularly targeted anti-ageing therapies that maintain health.

mTORC1 increases the rates at which numerous different mRNAs are translated, and a hallmark of $\mathrm{mTORC} 1$ inhibition is a reduction in overall protein synthesis ${ }^{2,3}$. Work in the model organisms $C$. elegans and Drosophila indicates that lifespan extension from mTORC1 inhibition is mediated in part through this global decrease in translation ${ }^{2,5,6}$. Furthermore, in $C$. elegans suppression of translation is sufficient to increase both lifespan and stress resistance ${ }^{7-12}$. A mechanistic understanding of how mRNA translation levels affect longevity should therefore provide mechanistic insights into how mTORC1 influences lifespan. 

which cells protect themselves from stressful conditions that include nutrient deprivation, and thermal-, oxidative-, and endoplasmic reticulum (ER) stress ${ }^{13,14}$. Those stresses induce the evolutionarily conserved ISR by activating kinases that phosphorylate and inhibit the translation initiation factor subunit elF2 $\alpha$, thereby imposing a broad reduction in cap-dependent mRNA translation ${ }^{13,14}$. This suppression of translation leads in turn to preferential translation of the activating transcription factor ATF4, which mobilizes stress defense mechanisms to reestablish homeostasis ${ }^{13,14}$. Although the ISR has important protective functions, its effects on longevity and health are complex. In $C$. elegans, genetic or pharmacological interventions that impair the ISR extend lifespan by inducing preferential translation of selective mRNAs ${ }^{15}$. In older mice, pharmacological ISR inhibition enhances memory and cognition by allowing protein synthesis to be maintained ${ }^{14,16}$. On the other hand, in S. cerevisiae the ATF4 ortholog Gcn4 promotes longevity ${ }^{17,18}$, and in $C$. elegans hexosamine pathway activation enhances proteostasis through the ISR and ATF- $4^{19}$. The effects of ATF4 on metazoan longevity have not been explored.

Here we have investigated whether and how ATF-4 affects lifespan in $C$. elegans. We find that ATF-4 but not upstream ISR signalling is required for longevity induced by conditions that inhibit protein synthesis. Importantly, ATF-4 is a prolongevity factor that extends lifespan when overexpressed on its own. ATF-4 increases lifespan not only by enhancing canonical anti-ageing mechanisms, but also by inducing transsulfuration enzyme-mediated $\mathrm{H}_{2} \mathrm{~S}$ production and therefore levels of protein persulfidation. Importantly, the anti-ageing benefits of mTORC1 suppression 
68 depend upon this ATF-4-induced increase in $\mathrm{H}_{2} \mathrm{~S}$ production, further supporting the

69 idea that they derive from lower translation rates and suggesting that increases in

70 ATF-4 and $\mathrm{H}_{2} \mathrm{~S}$ levels may recapitulate these benefits.

71 


\section{ATF-4 responds to translation suppression to increase $C$. elegans lifespan}

We investigated whether $C$. elegans atf- 4 is regulated similarly to mammalian

ATF4 at the level of mRNA translation. In mammals, 2-3 small upstream open reading frames (UORFs) within the ATF4 5' untranslated region (UTR) occupy the translation machinery under normal conditions, inhibiting translation of the downstream ATF4 coding region ${ }^{14,20}$. By contrast, when translation initiation is impaired by elF2 $\alpha$ phosphorylation, the last uORF is bypassed, and ATF4 is translated preferentially ${ }^{14,20}$. The C. elegans atf-4 ortholog (previously named atf-5) contains two 5' UTR uORFs (Fig. 1a; Extended Data Fig. 1a, 1b), deletion of which increases translation of a transgenic reporter ${ }^{21}$, predicting that translation of the atf-4 mRNA may be increased under conditions of global translation suppression.

We tested this idea in $C$. elegans that express green fluorescent protein (GFP) driven by the atf-4 upstream region, including the two uORFs (Patf-4(uORF)::GFP, Fig. 1a). Patf-4(UORF)::GFP expression was extremely low under unstressed conditions, but increased when translation was suppressed by treatment with the translation elongation blocker cycloheximide, or RNA interference (RNAi)-mediated knockdown of the tRNA synthase rars-1 (Fig. 1b, Extended Data Fig. 1c, 1d, 1e, 1f).

91 Compounds that induce ER stress and elicit the ISR, including tunicamycin (TM) or dithiothreitol (DTT), also strongly induced Patf-4(uORF)::GFP expression (Fig. 1b,

93 Extended Data Fig. 1c, 1d). By contrast, TM treatment increased atf-4 mRNA levels by only 1.5-fold (Fig. 1d, Extended Data Fig. 1g). Treatment with alpha-amanitin, which blocks transcription, prevented TM from increasing the levels of the atf- 4 mRNA but not Patf-4(uORF)::GFP fluorescence (Fig. 1c, 1d). Together, the data indicate that 
the Patf-4(uORF)::GFP reporter is regulated post-transcriptionally during ER stress. The endogenous atf-4 mRNA was expressed at steady levels during development and ageing (Extended Data Fig. 1h). By contrast, ribosome occupancy was decreased on its uORFs compared to the coding region as larval development progressed, with the

101 lowest levels apparent during the L4 stage after the body plan has been formed and growth has slowed (Fig. 1e, 1f). This suggests that the endogenous atf-4 gene is regulated translationally through its uORF region under unstressed conditions, possibly in response to growth-related cues. We conclude that, like mammalian ATF4, C. elegans atf-4 is regulated translationally and preferentially translated upon conditions of reduced protein synthesis (Extended Data Fig. 1i). synthesis. In C. elegans, RNAi-mediated knockdown of various translation initiation

111 factors increases lifespan ${ }^{7-12}$. Notably, the lifespan increases that occurred in 112 response to knockdown of ifg-1/elF4G, ife-2/elF4E, or eif-1A/elF1AY, was abrogated 113 in atf-4(tm4397) loss-of-function mutants (Fig. 2a, Supplementary Table 1). Similarly,

114 a low dose of cycloheximide extended the lifespan of wild type (WT) but not atf1154 (tm4397) animals (Fig. 2b, Supplementary Table 1). Thus, atf-4 is required for 116 longevity arising from a global reduction in protein synthesis.

The increase in ATF-4 translation that occurs during the canonical ISR is

119 induced by translation suppression that is imposed through increased elF2 $\alpha$ 120 phosphorylation ${ }^{22}$, but this might not be the case when translation is reduced by directly inhibiting translation initiation or elongation. Importantly, the low dose of 
cycloheximide that was sufficient to extend lifespan (Fig. 2b) increased ATF-4 expression (Extended Data Fig. 1f), but not elF2 $\alpha$ phosphorylation (Fig. 2c, 2d).

124 Similarly, depletion of the translation initiation factor ifg-1/elF4G reduced protein synthesis and dramatically increased expression of Patf-4(uORF)::GFP, but knocking down either ifg-1 or eif-1A only modestly increased elF2 $\alpha$ phosphorylation (Fig. 2e, 2f, expression without triggering canonical induction of the ISR through elF2 $\alpha$ phosphorylation.

We next investigated whether translation inhibition might increase lifespan independently of this canonical ISR signalling, using a well-characterized elF2 $\alpha$ mutant (eif2 $\alpha(q d 338))$ in which the serine at which inhibitory phosphorylation occurs

134 during the ISR (S49 in C. elegans; S51 in mammals) is mutated to phenylalanine, so that elF2 $\alpha$ phosphorylation and ISR induction are blocked ${ }^{23}$. A mutation that prevents phosphorylation of this serine partially suppresses lifespan extension from reduced insulin/IGF-1 signalling, suggesting that ISR signalling is important for longevity in this

138 context ${ }^{24}$. Importantly, the eif2 $\alpha(q d 338)$ mutation did not interfere with the atf-4dependent increase in lifespan that was seen with ifg-1 knockdown (Fig. 2j). We

140 conclude that canonical ISR signalling through elF2 $\alpha$ phosphorylation is not 141 necessarily required for translation inhibition to induce preferential ATF-4 translation or increase lifespan through ATF-4. 
SKN-1/NRF ${ }^{1,25}$. These evolutionarily conserved regulators are generally associated

with enhancement of protective mechanisms such as stress resistance, protein folding

149 or turnover, and immunity. To determine whether ATF-4 can actually promote

150 longevity, as opposed to being required generally for health, we investigated whether

151 an increase in ATF-4 levels might extend lifespan. Transgenic ATF-4-overexpressing

animals (ATF-4OE) exhibited nuclear accumulation of ATF-4 in neuronal, hypodermal,

and other somatic tissues under unstressed conditions (Patf-4::ATF-4(gDNA)::GFP;

154 Extended Data Fig. 2a). TM treatment doubled ATF-4 protein levels (Extended Data

155 Fig. 2b, Supplementary Data File 1), indicating that this ATF-4 transgene responds to

156 environmental stimuli. Importantly, ATF-4 overexpression increased lifespan by 7-

$15744 \%$ across > 10 independent trials, which included two experiments without 5-Fluoro-

158 2'deoxyuridine (FUdR) and analysis of two independent transgenic lines (Fig. 3a,

159 Supplementary Table 1). ATF-4 overexpression also prolonged healthspan (Fig. 3b,

160 Extended Data Fig. 2c, Supplementary Table 2). We conclude that the elevated

161 activity of the ATF-4 transcriptional program is sufficient to extend lifespan and promote health.

To identify longevity-promoting mechanisms that are enhanced by ATF-4, we used RNA sequencing (RNA-seq) to compare gene expression profiles in atf-4 loss-

166 of-function or ATF-4OE animals compared to WT under non-stressed conditions 167 (Extended Data Fig. 3a, 3b, Supplementary Table 3). Only a modest number of genes were detectably up- or down-regulated by atf-4 loss or overexpression, respectively

169 (Fig. 3c, Extended Data Fig. 3c, 3d). Notably, ATF-4 overexpression upregulated 170 several small heat shock protein (HSP) genes that are also controlled by HSF-1/HSF 171 (heat shock factor) and DAF-16/FOXO (Fig. 3d), and are typically induced by 
172 longevity-assurance pathways ${ }^{1}$. Each of the ATF-4-upregulated chaperone genes sip-1/CRYAA, hsp-70/HSPA1L, hsp-16.2/HSPB1, and $h s p-12.3 / H S P B 2$ was required

174 for lifespan extension from ATF-4 overexpression (Extended Data Fig. 3e;

175 Supplementary Table 1). Translation of atf-4 was increased within minutes by a heat

176 shock (Extended Data Fig. 3f), suggesting that ATF-4 functions in tandem with HSF-

177 1/HSF1. Together, the data suggest that ATF-4 enhances proteostasis mechanisms 178 that have been linked to longevity. 4 overexpression increased expression of the cytoprotective gene nit-1/Nitrilase (Fig. 3d), a canonical target of the xenobiotic response regulator $S K N-1 / N_{R}{ }^{25}$, along with expression of collagen genes that are typically upregulated by SKN-1/NRF in response to lifespan extension interventions (Fig. 3c) $)^{26}$. The 3kb predicted promoter regions of many ATF-4-upregulated genes included not only the binding consensus 186 for mammalian ATF4 (-TGATG-) ${ }^{27,28}$, but also sites for DAF-16, HSF-1, and SKN-1

187 (Fig. 3d, Supplementary Table 4, 5). Furthermore, many genes that were upregulated by ATF-4 overexpression had been detected in chromatin IP (ChIP) analyses of these transcription factors (Extended Data Fig. 3g, Supplementary Table 5). Each of those transcription factors is critical for lifespan extension arising from suppression of

191 translation ${ }^{10,11}$, and we determined that they are also needed for longevity conferred 192 by ATF-4 overexpression (Fig. 3e, Supplementary Table 1). ATF-4 overexpression also robustly upregulated two adenine nucleotide translocase genes (ANT; ant-1.3 and ant-1.4, Fig. 3c). The ANT complex is important for transport of ATP from the mitochondrial space into the cytoplasm, as well as for mitophagy ${ }^{29}$. Both ant-1.3 and 
197 Table 1). Together, our findings suggest that while the transcriptional impact of ATF-

1984 may seem limited in breadth, it cooperates with other longevity factors to enhance

199 the activity of multiple mechanisms that protect cellular functions, thereby driving

200 lifespan extension.

ATF-4 increases lifespan through $\mathrm{H}_{2} \mathrm{~S}$ production

To identify ATF-4-regulated genes that are conserved across species and might be particularly likely to have corresponding roles in humans, we queried our ATF-4OE vs WT RNA-seq results, and compared the top 200 significantly upregulated C. elegans genes against 152 mammalian genes that are thought to be regulated directly by ATF4 ${ }^{28}$. Seven orthologues of these genes were upregulated in ATF-4OE (Fig. 4a, Supplementary Table 4), four of which encoded components of the reverse transsulfuration (hereafter referred to as transsulfuration) pathway (cth-2/CTH), or associated mechanisms (glt-1/SLC1A2, C02D5.4/GSTO1, and F22F7.7/CHAC1) (Fig. 4b, Supplementary Table 4). The transsulfuration pathway provides a mechanism for utilising methionine to synthesize cysteine and glutathione when their levels are

213 limiting ${ }^{30}$, but the CTH enzyme (cystathionine gamma-lyase, also known as CGL or

214 CSE) also generates $\mathrm{H}_{2} \mathrm{~S}$ as a direct product. Underscoring the potential importance

215 of the $\mathrm{H}_{2} \mathrm{~S}$-generating enzyme CTH-2 for ATF-4 function, the levels of its mRNA and 216 protein were each increased by ATF-4 overexpression (Fig. 4c, 4d, 4e, Supplementary

217 Data File 1).

Regulation of amino acid biosynthesis genes is a conserved ATF4 function ${ }^{13}$, 220 and reduced levels of methionine ${ }^{31}$ and higher levels of $\mathrm{H}_{2} \mathrm{~S}^{32,33}$ have each been linked 221 to longevity. We did not detect any differences in the relative abundance of amino 
acids between ATF-4OE and WT animals (Supplementary Table 6), suggesting that

223 ATF-4 is unlikely to influence longevity by altering amino acid levels. By contrast,

224 ATF-4 overexpression consistently increased $\mathrm{H}_{2} \mathrm{~S}$ production capacity in a cth-2dependent manner (Fig. 4f, Extended Data Fig. 4a, 4b, 4c, 4d). Using the fluorescent $\mathrm{H}_{2} \mathrm{~S}$ probe MeRho-Az ${ }^{34}$, we also found that $\mathrm{H}_{2} \mathrm{~S}$ levels are reduced by mutation of either atf-4 or cth-2 (Fig. 4g). Taken together, our data indicate that ATF-4 promotes $\mathrm{H}_{2} \mathrm{~S}$ production by acting through $\mathrm{CTH}-2$. The increases in longevity and stress resistance that are conferred by ATF-4 overexpression are abrogated by cth-2 mutation or knockdown (Fig. 4h, Extended Data Fig. 4e, Supplementary Table 1, 7). Similarly, ifg-1/elF4G knockdown failed to extend lifespan in cth-2 mutant animals (Fig. 4i). We conclude that the increase in $\mathrm{H}_{2} \mathrm{~S}$ production that derived from $\mathrm{CTH}-2$ upregulation is a critical and beneficial aspect of ATF-4 function (Fig. 4j).

An important consequence of increased $\mathrm{H}_{2} \mathrm{~S}$ levels is an increase in the proportion of protein cysteine thiols (-SH) that are converted to persulfides (-SSH) ${ }^{35,36}$. Redox modification at reactive cysteine residues is critical in growth signalling and other mechanisms ${ }^{36}$, and in $C$. elegans thousands of redox-regulated cysteine residues are present in proteins that are involved in translation regulation, lipid and carbohydrate metabolism, stress signalling, and other fundamental biological processes $^{37}$. Under oxidising conditions, these thiols are readily converted to sulfenic acids $(-\mathrm{SOH})$, which is a reversible and in many cases regulatory modification that can proceed to irreversible and potentially damaging oxidized forms $\left(-\mathrm{SO}_{2} \mathrm{H},-\mathrm{SO}_{3} \mathrm{H}\right)$ (Fig.

$5 a)^{36}$. $\mathrm{H}_{2} \mathrm{~S}$ converts $-\mathrm{SOH}$ to $-\mathrm{SSH}$ in the process called persulfidation, preventing protein overoxidation and thereby preserving protein functions under stressed 246 conditions (Fig. 5a) ${ }^{35,36}$. The levels of overall protein persulfidation (PSSH) can be 
247 visualised in individual animals by using chemical probes and confocal microscopy ${ }^{35}$.

248 In C. elegans PSSH levels are decreased by mutation of the cth-2 paralog cth-1,

249 suggesting that they depend upon a background level of $\mathrm{H}_{2} \mathrm{~S}$ produced by the latter ${ }^{35}$.

250 We found that PSSH levels are also reduced by mutation of either cth-2 or atf-4, and

251 are modestly increased by ATF-4 overexpression (Fig. 5b, 5c). Taken together, our

252 results show that ATF-4 acts through multiple mechanisms to promote stress

253 resistance and longevity, and that a $\mathrm{CTH}$-2-driven increase in $\mathrm{H}_{2} \mathrm{~S}$ production and

254 persulfidation is an essential aspect of this program.

\section{A partial role for ATF-4 in some lifespan extension programs}

Given that atf-4 is required for lifespan extension in response to reduced translation, we investigated whether atf-4 and its transcriptional target cth-2 might be generally required for $C$. elegans longevity. Although ATF4/ATF-4 has been implicated in responses to mitochondrial stress or protein synthesis imbalance ${ }^{27,38}$, atf-

4 was dispensable for the increases in lifespan or oxidative stress resistance that

262 follow from developmental impairment of mitochondrial function (Extended Data Fig.

$2635 a, 5 b, 5 c$, Supplementary Table 1, 8). The extent of lifespan extension by reduced

264 insulin/IGF-1 signalling or germ cell proliferation was decreased by atf-4 mutation but

265 did not depend upon cth-2, consistent with other transsulfuration components and $\mathrm{H}_{2} \mathrm{~S}$

266 producers being implicated in the latter pathway (Extended Data Fig. 5d, 5e,

267 Supplementary Table 1$)^{39}$. The ATF-4-CTH-2 pathway of $\mathrm{H}_{2} \mathrm{~S}$ production may 268 therefore be fully essential specifically when lifespan extension is driven by suppression of protein synthesis. 
We also investigated whether the ATF-4-CTH pathway might be involved in

272 longevity induced by DR, which extends lifespan in essentially all eukaryotes. An

273 increase in $\mathrm{H}_{2} \mathrm{~S}$ production capacity has been implicated in mediating some DR

274 benefits in mammals ${ }^{33}$. In $C$. elegans, atf-4 was not required for lifespan to be

275 extended by a liquid culture food-dilution DR protocol but was partially required for

276 lifespan extension in the genetic DR-related model eat-2(ad1116) (Extended Data Fig.

277 5f, Supplementary Table 1). Consistent with these findings, transsulfuration pathway genes other than cth-2 are also partially required for eat-2 lifespan extension ${ }^{33,35}$, suggesting that in $C$. elegans other pathways than ATF-4-CTH might also increase

$280 \mathrm{H}_{2} \mathrm{~S}$ production during DR. In mammals, restriction of sulfur-containing amino acids

281 (methionine and cysteine) acts through ATF4 and CTH to boost endothelial $\mathrm{H}_{2} \mathrm{~S}$ levels and angiogenesis ${ }^{47}$, and multiple longevity interventions increase CTH mRNA levels ${ }^{48}$, suggesting a possible role for the ATF4-CTH pathway in DR. Supporting this idea, our bioinformatic analysis revealed that CTH mRNA levels increased in various mouse tissues in response to DR (32/36 profiles), rapamycin (4/6 profiles), or growth hormone insufficiency (8/8 profiles) (Extended Data Fig. 5g, Supplementary Table 11).

287 Therefore, ATF4-induced $\mathrm{H}_{2} \mathrm{~S}$ upregulation is likely to be evolutionarily conserved as a contributor to lifespan extension.

\section{Longevity from mTORC1 suppression is driven by ATF-4 and $\mathrm{H}_{2} \mathrm{~S}$}

Because mTORC1 inhibition increases lifespan in part by reducing protein synthesis ${ }^{2,5,6}$, we hypothesized that ATF-4 might be activated and required for lifespan extension when mTORC1 is inhibited. The heterodimeric RAG GTPases transduce amino acid signals to activate mTORC1 signalling, and are composed of RAGA-1 and

RAGC-1 in C. elegans. mTORC1 is required for $C$. elegans larval development ${ }^{2}$, but 
296 lifespan can be increased by RNAi-mediated knockdown of either raga-1 or ragc-1

297 during adulthood, or by a partial loss-of-function mutation of raga-12,3. The former

298 strategy allows mTORC1 activity to be reduced without any associated developmental

299 effects. Adulthood RAG gene knockdown reduced protein synthesis (Fig. $2 \mathrm{~g}$ and $2 \mathrm{~h}$ ),

300 consistent with previous studies of mTORC $1^{2,3}$, but did not induce elF $2 \alpha$

301 phosphorylation (Fig. $2 \mathrm{e}$ and 2f). Under these conditions Patf-4(uORF)::GFP

302 expression was increased robustly even when elF2 $\alpha$ phosphorylation was blocked

303 genetically (Fig. 6a, 6b, Supplementary Table 9). We conclude that ATF-4 is

304 preferentially translated when mTORC1 activity is reduced and translation rates are

305 low, and that this occurs independently of the canonical ISR mechanism of increased

306 elF2 $\alpha$ phosphorylation.

Importantly, the increases in lifespan extension, stress tolerance, and

healthspan that resulted from loss of either raga-1 or ragc-1 function required atf-4 but

310 not phosphorylation of elF2 $\alpha$ (Fig. 6c, 6d, 6e, 6f, Extended Data Fig. 2c, 5c, 6a,

311 Supplementary Table 1-2, 7, 8, 10), consistent with our analyses of Patf-

$3124(u O R F)::$ GFP activation. In a single trial, the longevity induced by raga-1 knockdown

313 was blunted by ATF-4 overexpression, suggesting that too much ATF-4 activity might

314 be harmful (Extended Data Fig. 2c), but this has been observed for some other pro-

315 longevity factors ${ }^{25}$. In summary, the data demonstrate that ATF-4 activation plays an essential role in the benefits of reducing mTORC1 activity

319 lifespan, we were surprised to find that atf-4 was dispensable for lifespan extension

320 from rapamycin treatment, even though rapamycin increased ATF-4 translational 
321 reporter expression (Fig. 6g, Extended Data Fig. 6b, Supplementary Table 1, 9).

322 Notably, mTOR is present in both the mTORC1 and mTORC2 complexes (Extended

323 Data Fig. 6c) ${ }^{3}$. mTORC2 is not as well understood as mTORC1, but it functions in

324 growth signalling and its activation involves binding to the ribosome, suggesting an

325 association with translation regulation (Extended Data Fig. 6c) ${ }^{40}$. Rapamycin mechanistically inhibits mTORC1 activity, but continuous rapamycin treatment also reduces mTORC2 activity by blocking complex assembly ${ }^{41,42}$, leading us to investigate the possible involvement of atf-4 in mTORC2 effects. Knockdown of the essential mTORC2 subunit RICT-1 (Rictor) suppressed translation (Fig. 2g, 2h) and increased

330 Patf-4(uORF)::GFP expression independently of elF2 $\alpha$ phosphorylation (Fig. 2e, $2 f$,

331 6b, Supplementary Table 9). The effects of mTORC2 on C. elegans lifespan are complex, but adulthood RNAi knockdown of rict-1 extends lifespan ${ }^{2,6}$. Lifespan extension by rict-1 knockdown required atf-4 (Fig. 6h, Supplementary Table 1). 334 Interestingly, however, simultaneous inactivation of mTORC1 and mTORC2 by knocking down both raga-1 and rict-1 extended lifespan independently of atf-4 (Fig.

6i, Supplementary Table 1), as occurred with rapamycin treatment, suggesting that when the activity of both mTOR complexes is suppressed the requirement for atf- 4 is 338 relieved.

We investigated whether an ATF-4-mediated increase in $\mathrm{H}_{2} \mathrm{~S}$ production is

341 required for lifespan extension arising from inhibition of the mTORC1 or mTORC2

342 kinase complexes. Genetic inhibition of either mTORC1 or mTORC2 increased $\mathrm{H}_{2} \mathrm{~S}$

343 production capability in an atf-4-dependent manner (Fig. 7a, 7b, Extended Data Fig.

$3446 \mathrm{~d}, 6 \mathrm{e})$. Furthermore, the ATF-4 target gene cth-2 was required for the increased

345 lifespan of animals with reduced mTORC1 or mTORC2 activity (Fig. 7c, 7d,

346 Supplementary Table 1, 7), suggesting that an ATF-4/CTH-2-mediated increase in 
$\mathrm{H}_{2} \mathrm{~S}$ production is essential for the benefits of either mTORC1 or mTORC2 inhibition.

348 Knockdown of cth-2 prevented mTORC1 inhibition from increasing stress resistance,

349 further supporting this idea (Extended data Fig. 6f). Interestingly, simultaneous

350 knockdown of raga-1 and rict-1 dramatically increased $\mathrm{H}_{2} \mathrm{~S}$ production capability, and

351 this was greatly reduced but not eliminated in the atf-4 loss-of-function mutant

352 (Extended Data Fig. 6g). This result, together with the dispensable role for ATF-4 in longevity under these conditions, suggests that simultaneous inhibition of both mTOR complexes mobilizes mechanisms that increase lifespan independently of atf-4 and possibly $\mathrm{H}_{2} \mathrm{~S}$. response to interventions that boost $\mathrm{H}_{2} \mathrm{~S}$ production through this pathway, including

360 mTORC1 inhibition. Accordingly, in raga-1 mutant adults PSSH levels were elevated

361 in an atf-4-dependent manner (Fig. 7e, 7f). Taken together, our results show that

362 reduced $\mathrm{mTORC} 1$ signalling leads to preferential translation of ATF-4, which acts 363 through $\mathrm{CTH}-2$ to promote stress resilience and healthy ageing by increasing $\mathrm{H}_{2} \mathrm{~S}$ 364 production, and possibly through the resulting increase in PSSH levels across the 365 proteome (Fig. 7g). 


\section{Discussion}

We have identified ATF-4, the transcriptional effector of the ISR, as a pro-

369 longevity factor that can extend $C$. elegans lifespan when overexpressed.

370 Furthermore, conditions that reduce mRNA translation, including mTORC1 inhibition,

371 increase ATF-4 expression without activating canonical ISR signalling that is

372 downstream of elF2 $\alpha$ phosphorylation, and require ATF-4 for lifespan extension.

373 Previous studies revealed that longevity arising from inhibition of translation depends upon preferential translation of protective genes ${ }^{43}$ and increased transcription of stress defence genes ${ }^{6,10}$. Our new findings link these mechanisms by revealing that preferentially translated ATF-4 cooperates with DAF-16/FOXO, HSF-1/HSF, and SKN-1/NRF to drive protective gene transcription. Viewed alongside the welldocumented pro-longevity activity of $S$. cerevisiae Gcn4 (ATF-4 ortholog) ${ }^{17,18}$ and evidence that ATF-4 levels and activity are increased in long-lived mouse models ${ }^{44,45}$, our results indicate that ATF-4 has an ancient and broadly conserved function in promoting longevity.

382

A striking aspect of our findings is that the longevity and health benefits of ATF-

4 depend upon activation of its target transsulfuration pathway gene cth-2, and the resultant increase in $\mathrm{H}_{2} \mathrm{~S}$ production. For many years an understanding of how $\mathrm{H}_{2} \mathrm{~S}$ might promote health and longevity proved to be elusive ${ }^{46}$, but recent work has

387 implicated increased PSSH and its salutary effects on the proteome ${ }^{35}$. This modification protects the proteome from the effects of oxidative stress by "rescuing" sulfenylated cysteine residues from the fate of further oxidation (Fig. 5a), declines

390 during ageing, and is increased in other long-lived models ${ }^{35}$. Here we demonstrated

391 that PSSH levels were increased or decreased by ATF-4 overexpression or cth-2 
mutation, respectively, and that mTORC1 inhibition increased PSSH levels through

393 ATF-4 (Figs. 5 and 7). These results provide the first definition of a regulatory pathway

394 through which a pro-longevity intervention increases $\mathrm{H}_{2} \mathrm{~S}$ and PSSH levels (Fig. 7g).

395 Although persulfides can be introduced during translation ${ }^{47}$, our results align with

396 previous evidence that $\mathrm{H}_{2} \mathrm{~S}$ levels may largely determine the extent of this protective

397 cysteine modification ${ }^{35}$. It was particularly intriguing that the ATF-4/CTH-2 pathway, which boosts $\mathrm{H}_{2} \mathrm{~S}$ formation and $\mathrm{PSSH}$ levels, was required for lifespan extension from mTORC1 suppression. This ATF-4/H2S-induced posttranslational shift in PSSH levels could influence many biological functions, including the activity of redox-regulated signalling pathways ${ }^{35,37}$, making it of interest to elucidate how these modifications mediate the downstream effects of mTORC1 signalling.

Our data add to the evidence that translation suppression is an essential effector of the longevity effects of $\mathrm{mTORC} 1$ inhibition ${ }^{5,6}$. We were surprised, however, to find that mTORC2 knockdown decreased protein synthesis levels and depended upon ATF-4 for lifespan extension (Figs. 2 and 6). It will be very interesting to elucidate the mechanisms underlying the former observation. It was also surprising that either simultaneous mTORC1 and mTORC2 inhibition or rapamycin extended lifespan independently of ATF-4, suggesting that when both mTOR complexes are inhibited an

411 independent mechanism compensates for lack of ATF-4. An understanding of how 412 this occurs is likely to identify additional mechanisms that promote longevity.

Our evidence that reduced mTORC1 activity promotes longevity by increasing

415 ATF-4 levels contrasts with mammalian evidence that pharmacological mTORC1 416 inhibition reduces ATF4 translation ${ }^{20,48}$. However, those findings were obtained in 
cultured cells in which mTORC1 activity was elevated genetically or by growth factor

418 treatment, a very different scenario from adult $C$. elegans in vivo, in which growth has

419 largely ceased and most tissues are post-mitotic. It seems logical that mTORC1 might 420 increase ATF4 translation under the former conditions, given the importance of 421 mTORC1 for translation of many genes and the need to maintain amino acid levels under conditions of high growth activity. Importantly, it is consistent with our $C$. elegans results that analyses of mouse liver found that ATF4 protein levels and activity are increased in long-lived models that include rapamycin treatment and nutrient restriction ${ }^{44,45}$, and that $\mathrm{mTORC1}$ hyperactivation (TSC1 deletion) decreases $\mathrm{CTH}$ expression and prevents DR from increasing $C T H$ mRNA levels ${ }^{33}$. It will be interesting in the future to determine how mammalian mTORC1 influences ATF4 in vivo under different conditions, including analysis of tissues with varying rates of growth and levels of mTORC1 activity.

Although inhibition of $\mathrm{mTORC} 1$ has received widespread enthusiasm as an anti-ageing strategy, mTORC1 maintains fundamental processes that include protein

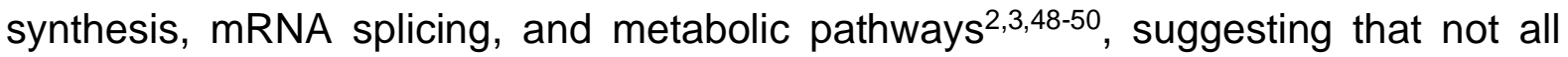
effects of mTORC1 suppression are necessarily beneficial. Similarly, although conditions that suppress ISR signalling can promote longevity through effects on selective translation ${ }^{15}$, ISR signalling through elF2 $\alpha$ contributes to lifespan extension 437 from reduced insulin/IGF-1 signalling ${ }^{24}$, and our results demonstrate that the downstream ISR effector ATF-4 is a potent pro-longevity factor. Furthermore, while pharmacological ISR inhibition preserves cognitive functions during ageing by 440 maintaining protein synthesis ${ }^{14,16}$, our findings suggest that ISR suppression could 441 reduce levels of $\mathrm{H}_{2} \mathrm{~S}$, which has been shown to prevent neurodegeneration ${ }^{51,52}$. In 
442 these and other settings, targeted mobilisation of beneficial mechanisms that are

443 activated by ATF-4, including $\mathrm{H}_{2} \mathrm{~S}$ production, might be of promising long-term value.

444 Consistent with this notion, $\mathrm{H}_{2} \mathrm{~S}$ confers many cardiovascular benefits in mammals,

445 including a reduction in blood pressure ${ }^{52,53}$, and patients suffering from vascular

446 diseases show reduced $\mathrm{CTH}$ and $\mathrm{H}_{2} \mathrm{~S}$ levels ${ }^{54}$, prompting clinical trials of $\mathrm{H}_{2} \mathrm{~S}$ -

447 releasing agents for cardiovascular conditions (NCT02899364 and NCT02278276). It

448 could be of considerable value to examine the potential benefits of ATF4 and $\mathrm{H}_{2} \mathrm{~S}$ in

449 various settings, including prevention of ageing-related phenotypes and disease. 


\section{Author contributions}

C.Y.E. and T.K.B. conceived the study and designed the experiments. All authors participated in analysing and interpreting the data. C.Y.E., K.P., M.B., S.R.S., C.S., and R.V. performed lifespan assays. C.Y.E., M.B., C.S., and R.V. performed oxidative stress assays. C.Y.E., M.B., and C.S. performed thermotolerance assays. C.Y.E., M.B., K.P., S.R.S., and R.V. scored GFP reporters. C.S., R.E., J.M., A.L., and D.P. performed $\mathrm{H}_{2} \mathrm{~S}$ measurement assays. P.L. and C.H. performed Ribo-sequencing analysis. D.P. and M.F. performed persulfidation assays and analysis. C.S. and C.Y.E. analyzed transcription profiles. C.Y.E., C.S., and J.M. performed qRT-PCR. J.M. and

R.V. performed the Western bots and puromycin assays. I.M. and W.B.M. generated transgenic strains. C.Y.E. performed all other assays. C.Y.E., T.K.B., and J. M. wrote the manuscript in consultation with the other authors.

\section{Competing interests}

The authors have no competing interests to declare. Correspondence should be addressed to C. Y. E. and T.K.B.

\section{Data availability}

469 The RNA sequencing data in this publication have been deposited in NCBl's Gene

470 Expression Omnibus and are accessible through GEO Series accession number GSE173799.

\section{Acknowledgement}

474 We thank Alex Hofer, Anita Goyala, Sara Schütze, Carolin Imse, Julia Rogers, and

475 Lorenza E. Moronetti Mazzeo for help with scoring lifespan, stress, and GFP assays, 
476 Michael Steinbaugh for help with the initial analysis of the RNA sequencing data,

477 Stephanie Lin for contributing to earlier stages of this work, S. Mitani and the National

478 BioResource Project for the atf-4(tm4212 and tm4397) alleles, Mike Crowder for the

479 rars-1(gc47) allele, Chi Yun and David Ron for the Patf-4(uORF)::GFP reporter strain,

480 WormBase for curated gene and phenotype information, Jay Mitchell and Nancy Pohl

481 for comments on the manuscript, and Spalentor and Michael Hall for inspiration. Some

482 strains were provided by the CGC, which is funded by the NIH Office of Research

483 Infrastructure Programs (P40 OD010440). Portions of this research were conducted

484 on the Orchestra High Performance Computer Cluster at Harvard Medical School

485 (NCRR 1S10RR028832-01). Supported by funding from the Swiss National Science

486 Foundation PBSKP3_140135, P300P3_154633, and PP00P3_163898 to C.Y.E. and

487 C.S., and PZ00P3-185927 to A.L., the Leenaards and Novartis Foundation to A.L.,

488 ETH Research Grant (ETH-30-16-2) to R.V., the lacocca Family Foundation to J.M., 489 and NIH R35GM122610 and AG054215 to T.K.B. Part of this research was conducted 490 while Collin Y. Ewald was an Ellison Medical Foundation/AFAR Postdoctoral Fellow. 
492 Figure Legends

493

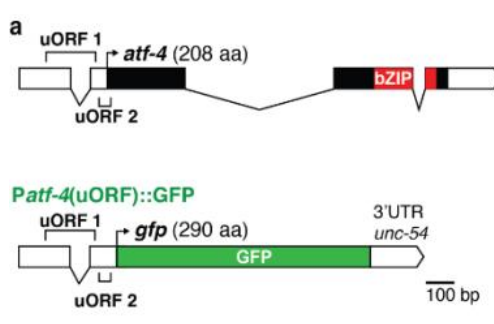

b Patf-4(UORF)::GFP
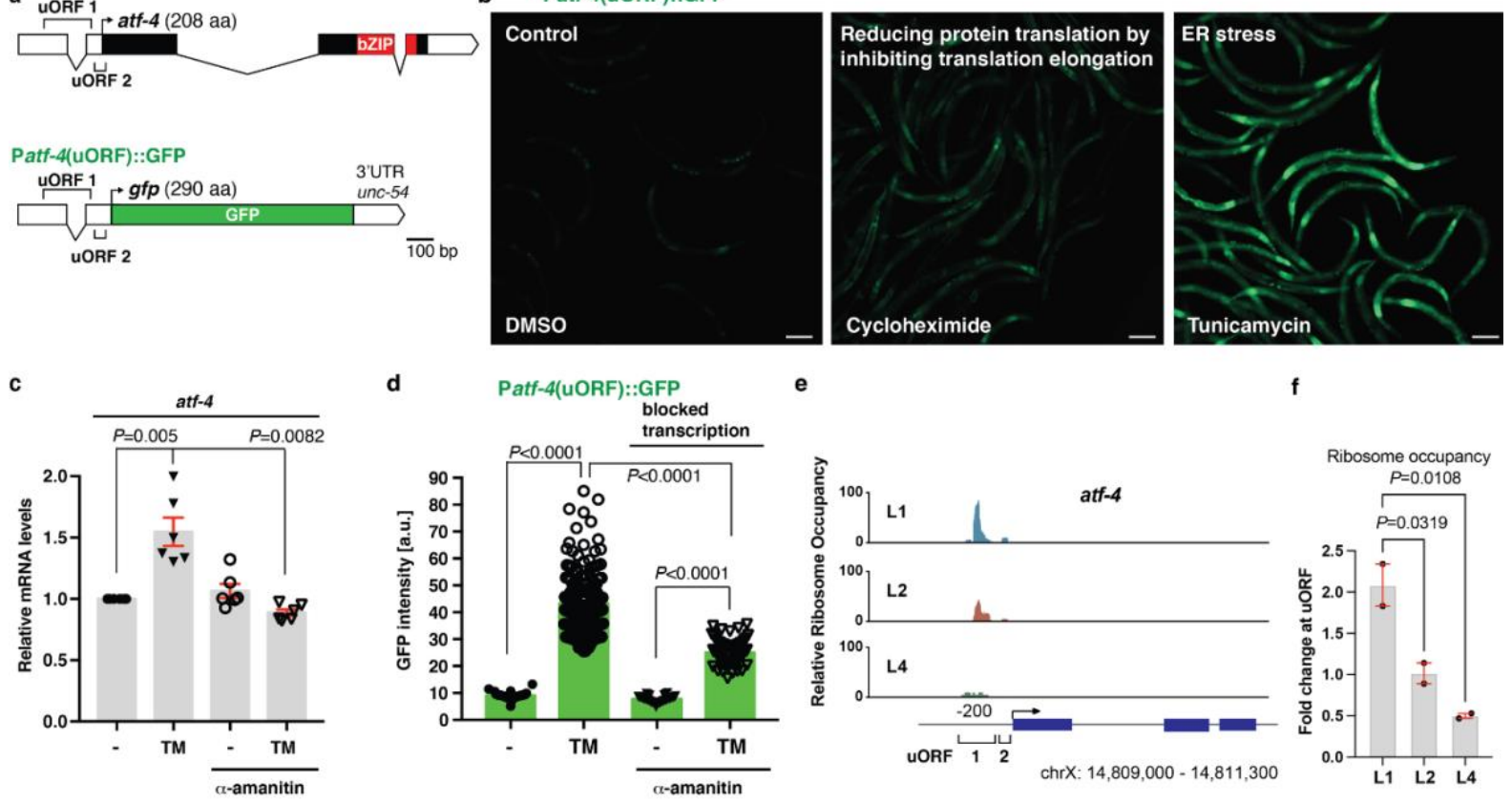

d Patf-4(UORF)::GFP e
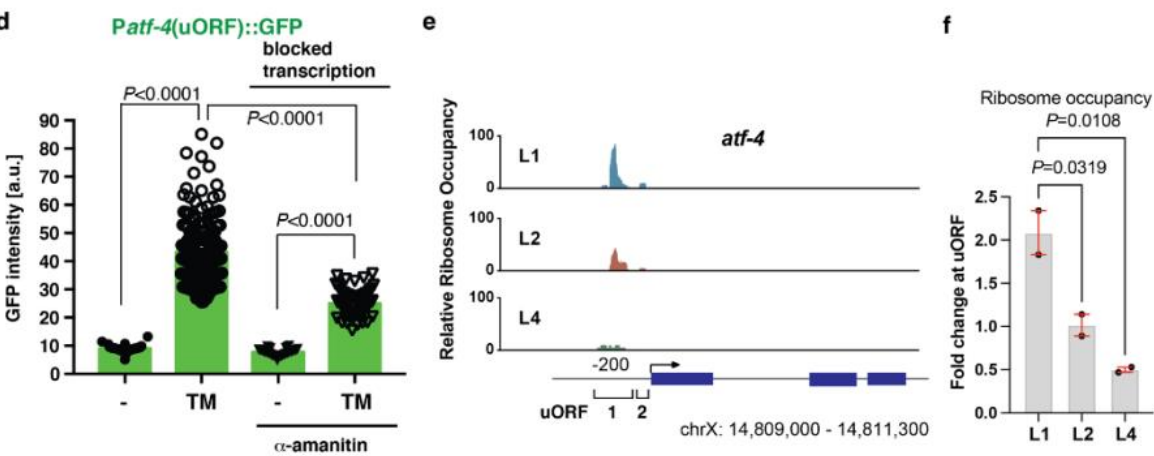

protein synthesis. a Schematic diagram of the atf-4 mRNA and the Patf4(UORF)::GFP reporter. UTRs are represented as empty boxes, exons as filled boxes, and the basic leucine zipper domain (bZIP) in red. b Representative images showing that reducing translation by administering $7.2 \mathrm{mM}$ cycloheximide for 1 hour or $35 \mu \mathrm{g} / \mathrm{ml}$ tunicamycin (TM) for 4 hours increased expression of transgenic Patf-4(uORF)::GFP

501 in L4 stage animals. Quantification of GFP fluorescence intensity is shown in Extended

502 Data Fig. 1c. Scale bar $=100 \mu \mathrm{m}$. c A 1 hour pre-treatment with $0.7 \mu \mathrm{g} / \mathrm{ml} \alpha$-amanitin

503 (RNA Pol II inhibitor) prevented 4 hours of $35 \mu \mathrm{g} / \mathrm{ml}$ TM treatment from increasing atf-

5044 mRNA levels in L4 stage animals. Mean \pm SEM. Three independent trials, measured

505 in duplicates. $P$ values are relative to WT (N2) determined by one sample $t$-test, two-

506 tailed, hypothetical mean of $1 . \mathrm{d} \mathrm{A} 1$ hour pre-treatment with $0.7 \mu \mathrm{g} / \mathrm{ml} \alpha$-amanitin did 507 not prevent TM treatment from increasing levels of transgenic Patf-4(uORF)::GFP expression in L4 stage animals. Mean \pm SEM. $n>30$ animals, 2 independent trials, 
509 One-way ANOVA with post hoc Tukey. e Stage-specific ribosome occupancy profiles

510 of the endogenous atf-4 mRNA, along with quantification of relative uORF occupancy

511 (f). Analysis of ribosomal profiling data ${ }^{55}$ revealed a decrease in ribosome occupancy

512 on the endogenous atf-4 uORFs under unstressed conditions during late larval

513 development. Occupancy profiles were generated by assigning counts to the atf-4

514 transcript based on the number of raw reads at each position. Blue boxes indicate the

515 atf-4 exons. One-way ANOVA post hoc Dunnett's test.

516 
ATF-4 [C. elegans] ATF5 [human]

cons

ATF-4 [C. elegans] ATF5 [human]

cons

ATF-4 [C. elegans] ATF 4 [human]

cons

ATF-4 [C. elegans] ATF 4 [human]

cons

ATF-4 [C. elegans]

ATF5 [human]

cons

ATF-4 [C.elegans] ATF5 [human]

cons

ATF-4 [C. elegans] ATF 4 [human]

cons

ATF-4 [C. elegans] ATF5 [human]
ATF 4 [human]

ATF 4

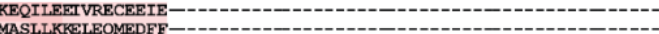

MPDDLLTTLDDTCDLFAPLVQETNKQEPQTVNPIGHIPESLTKPDQVAPFTFLQPLPLSPGVI
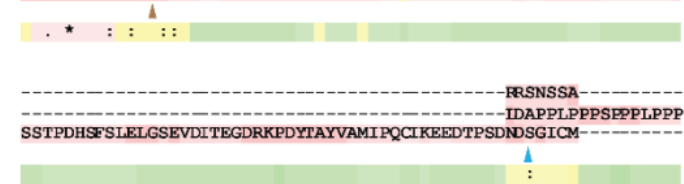

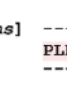

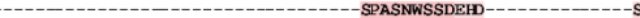
PLPPAPSLPLSLPSEDIPQPPVLDTLILLAIYCRNEAGQEEVGMPPIPPPQ-QPPPESPPQPS

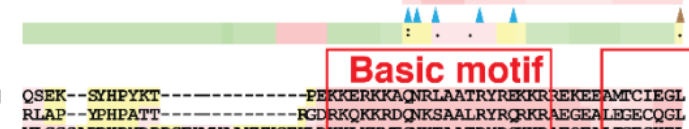

QSEK--SYHPYKT

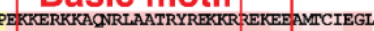

VLCGSARPKPYDPPGERMVAAKVKGEKLDKKKLKKMMEGNKTAATRYROKKRAEQEAALTEECKEI

\section{eucine-zipper}

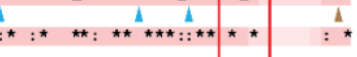

cons

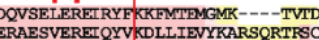

EKKNEAIKERADSLAKEIQYLKDLIEEVRKARGKKRV-D

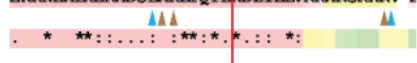
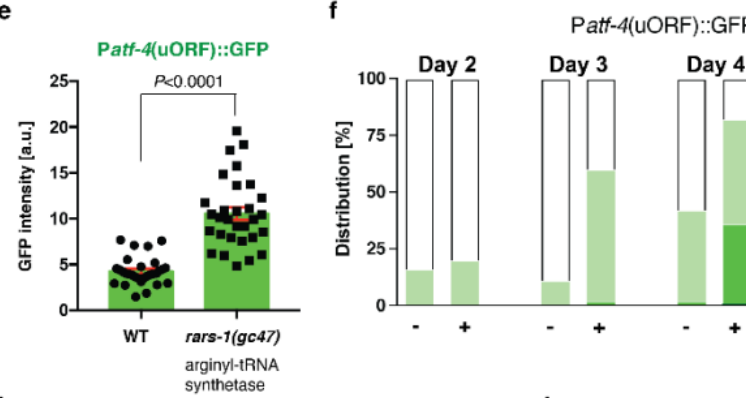

Patt-4(UORF)::GFP
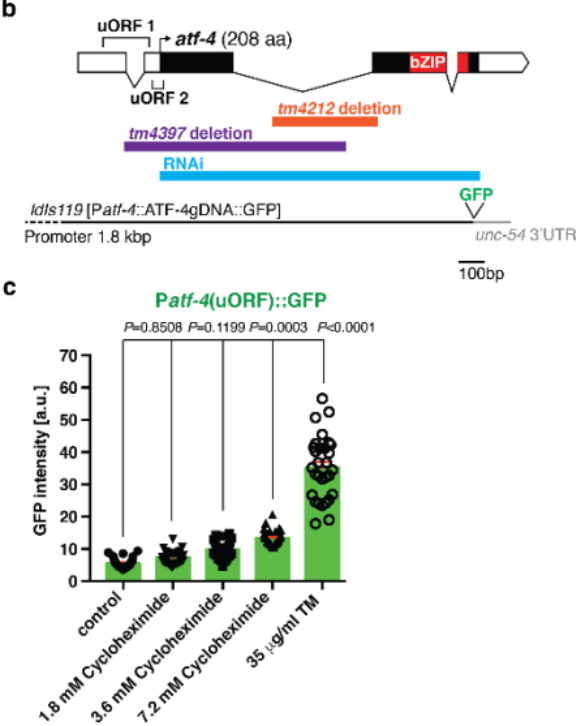

d
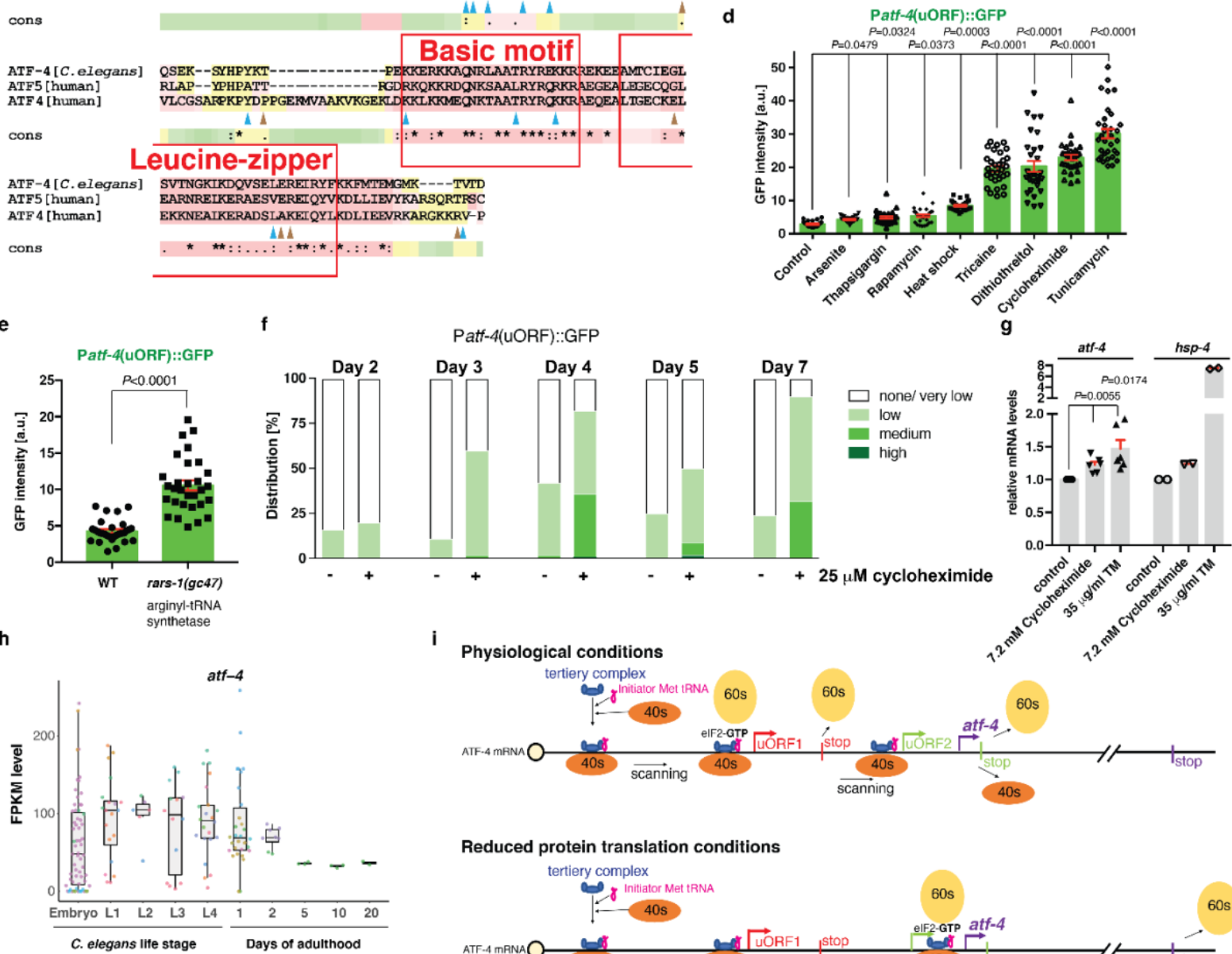

Reduced protein translation conditions

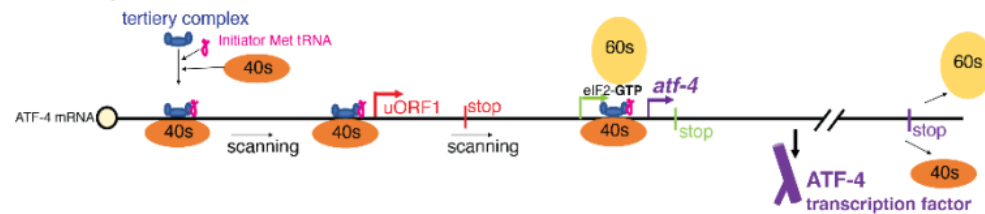

518 Extended Data Fig. 1. Translational regulation of $C$. elegans atf-4 expression. a

519 C. elegans ATF-4 (T04C10.4) shares identical amino acids with human ATF4 (blue 
521 similar to that of human ATF4. Stars indicate identical amino acids among C. elegans 522 ATF-4, human ATF4, and ATF5 (25 in total). Single dots indicate that size or

523 hydropathy is conserved, while double dots indicate that both size and hydropathy are

524 conserved between the corresponding residues. The $C$. elegans ortholog of human

525 ATF5 is ATFS156. b Diagram of atf-4 mRNA, mutations and RNAi clone, and the Patf-

$5264(u O R F):: G F P$ transgene. The atf-4 mRNA has an extensive 5'UTR of 250 nucleotides

527 containing two uORFs, of which uORF1 translates into a 39 amino acids (aa) peptide and UORF2 into a 14 aa peptide. The tm4397 variation is an 806 base pair (bp) deletion that covers part of the uORF1, the uORF2, the translational start site and the

530 first exon, suggesting that tm4397 is a putative null allele. c Quantification of GFP

531 fluorescence in Patf-4(uORF)::GFP transgenic animals at the L4 stage treated either with cycloheximide for 1 hour or TM for 4 hours. Mean \pm SEM. One-way ANOVA with post hoc Tukey. d Quantification of GFP fluorescence showing the effects on ATF-4 expression of various drug treatments or interventions that reduce mRNA translation. L4 stage animals were treated either with $20 \mathrm{mM}$ arsenite (an inducer of oxidative stress) for $30 \mathrm{~min}, 200 \mathrm{mM}$ thapsigargin (which induces ER stress by inhibiting the ER $\mathrm{Ca}^{2+}$ ATPase) for 4 hours, $100 \mu \mathrm{M}$ rapamycin (an inhibitor of mTORC1) for overnight, heat shock at $35^{\circ} \mathrm{C}$ for $30 \mathrm{~min}, 2 \%$ tricaine for 1 hour (which induces ER stress), $10 \mathrm{mM}$ dithiothreitol (which induces reductive ER stress) for 4 hours, $10 \mathrm{mM}$ cycloheximide (an inhibitor of translation elongation) for 1 hour, or $35 \mu \mathrm{g} / \mathrm{ml}$ tunicamycin (a glycosylation inhibitor that induces ER stress) for 4 hours. Mean \pm

542 SEM. One-way ANOVA with post hoc Tukey. e Nonsense mutation in the arginyl-tRNA 543 synthetase rars-1(gc47) increased Patf-4(uORF)::GFP expression compared to WT at 544 the L4 stage. Mean \pm SEM. Unpaired two-tailed Student's $t$-test, hypothetical mean of 545 1. f Quantification of GFP intensity in transgenic Patf-4(uORF)::GFP animals at 
546 different ages showing that $25 \mathrm{uM}$ cycloheximide induced ATF-4 induction. $\mathrm{n}=2$

547 independent trials. L4 animals were transferred onto plates containing either DMSO

548 or cyclohexmide with FUdR. g Quantification of atf-4 mRNA levels after cycloheximide

549 or TM treatment in L4 stage animals. $\mathrm{n}=3$ independent trials, measured in duplicates.

550 In one trial, $h s p-4$ mRNA was assessed as a positive control for ER stress. Mean \pm

551 SEM. $P$ values relative to control determined by one-sample $t$-test, two-tailed, a

hypothetical mean of 1. h Expression levels of atf-4 mRNA plotted as Fragments Per

Kilobase of transcript per Million mapped reads (FPKM) during development and ageing. The atf-4 mRNA expression levels of untreated WT $C$. elegans were retrieved using the RNAseq FPKM Gene Search tool (www.wormbase.org). The boxplots represent the overall expression pattern and the color of the individual dots refer to the 32 individual studies used. Hypothetical working model for $C$. elegans atf-4 preferential translation, assuming that its regulation is the same as mammalian ATF $4^{14,20}$. The $C$. elegans atf-4 gene encodes two uORFs. After translating the first

560 uORF, the small ribosomal subunit will continue scanning along the ATF4 mRNA.

561 Under non-stressed conditions, i.e., when high amounts of the elF2-GFP bound Met-

$562 \mathrm{tRNA}_{\mathrm{i}}{ }^{\text {Met }}$ are available, the small ribosomal subunit will readily acquire the elF2 ternary complex, and the large ribosomal subunit will associate to translate the second uORF.

564 In mammalian ATF4, the ribosome disassociates from the atf-4 mRNA after translating

565 the last uORF. However, under stress or reduced translational conditions, i.e., low 566 amounts of the elF2-GFP bound Met-tRNAi ${ }^{\text {Met }}$ availability, the association of the large

567 to the small ribosomal subunit is delayed, whereby the inhibitory second uORF is 568 skipped and the re-initiation complex starts to translate the ATF-4 coding region.

569 Phosphorylation of elF2 $\alpha$ subunit inhibits the guanine nucleotide exchange factor 
bioRxiv preprint doi: https://doi.org/10.1101/2020.11.02.364703; this version posted August 24,2021 . The copyright holder for this preprint

(which was not certified by peer review) is the author/funder, who has granted bioRxiv a license to display the preprint in perpetuity. It is made available under aCC-BY-NC-ND 4.0 International license.

570 elF2B, which lowers the exchange of the elF2-GDP to elF2-GTP and thereby lowers

571 global mRNA translation initiation. 

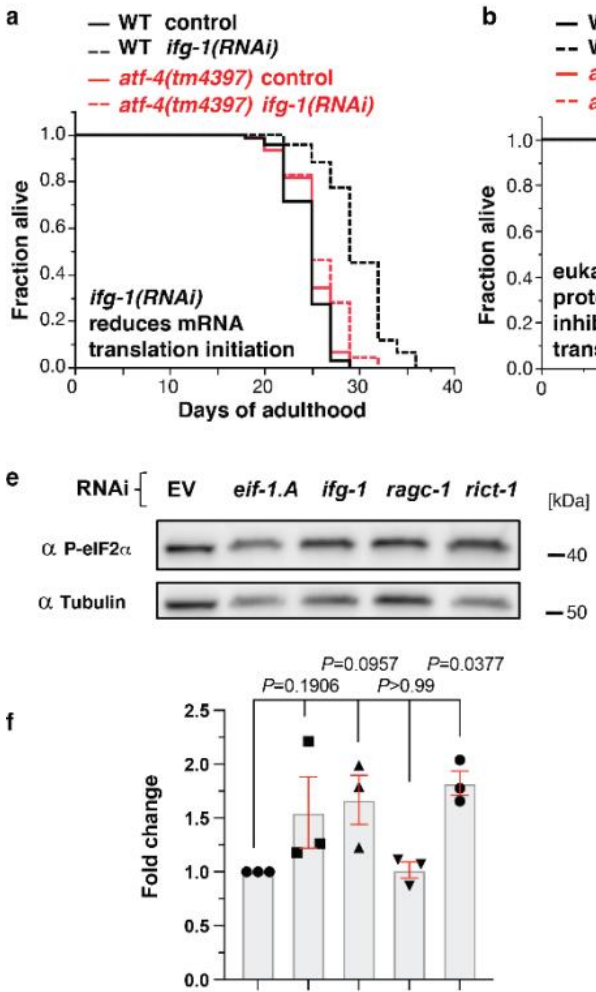

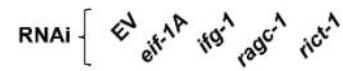

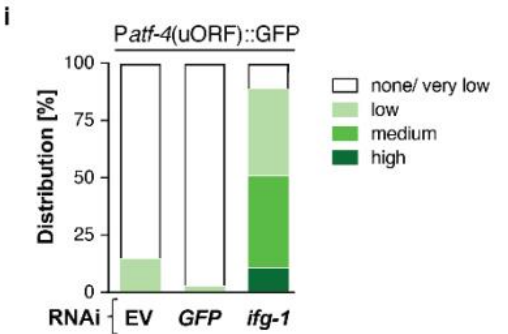

j b - WT control

-- WT $25 \mu \mathrm{M}$ cycloheximide

- atf-4(tm4397) control

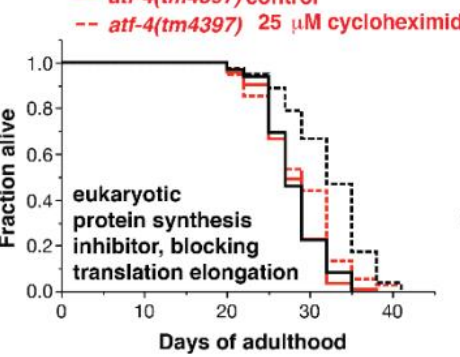

g

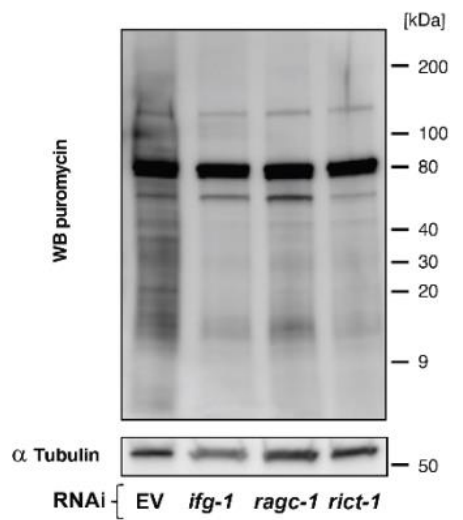

h
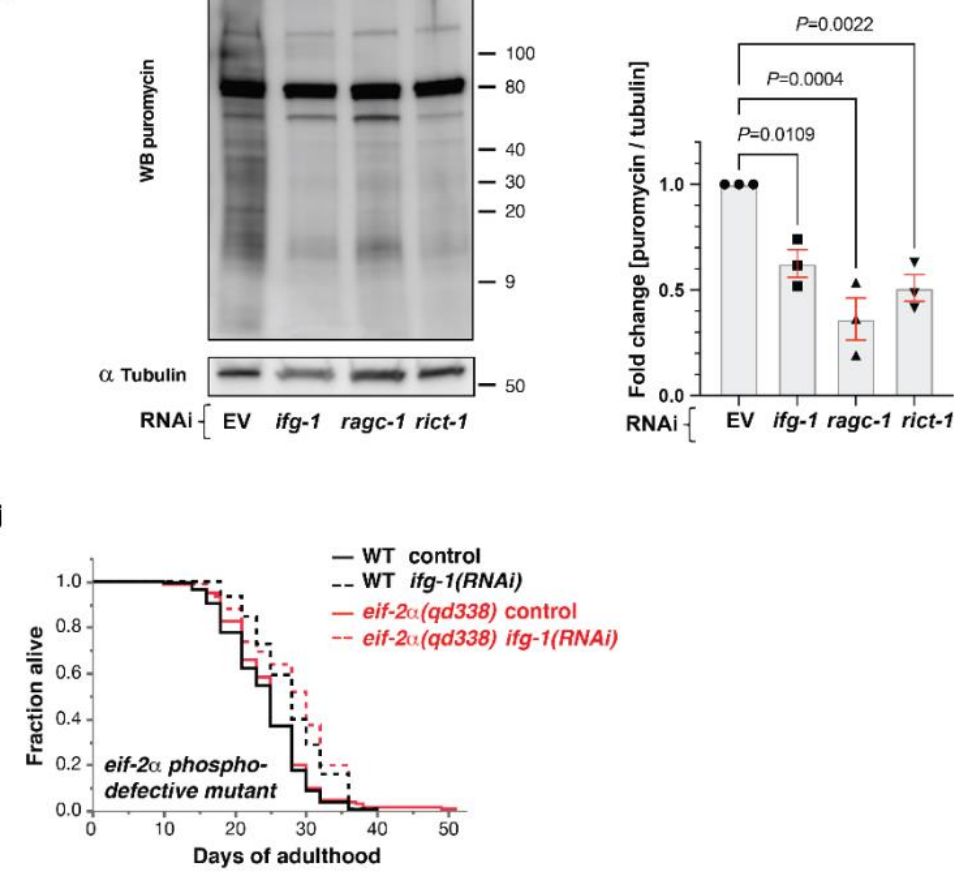

Figure 2. ATF-4 mediates lifespan extension from translation inhibition. a Adult-

577 that treatment with $35 \mu \mathrm{g} / \mathrm{ml}$ tunicamycin for 4 hours dramatically increased elF2 $\alpha$

578 phosphorylation levels in L4 stage animals, while treatment with $7.2 \mathrm{mM}$ 579 cycloheximide for 1 hour did not. One-way ANOVA with post hoc Tukey. e

580 Representative western blots and quantification (f) showing the effects of adult-

581 specific knockdown of eif-1.A, ifg-1, ragc-1, or rict-1 on elF2 $\alpha$ phosphorylation levels. 
582 One-way ANOVA with Dunnetts's post-test compared to EV. g Representative

583 western blots of puromycin incorporation assay and quantification (h) showing that

584 adult-specific knockdown of ifg-1, ragc-1, or rict-1 decreased translation. One-way

585 ANOVA with Bonferroni post-test. i Quantification of GFP fluorescence showing that

586 adult-specific ifg-1 knockdown increases expression of Patf-4(uORF)::GFP. j Adult-

587 specific knockdown of ifg-1 comparably extended the lifespan of WT animals and eif-

$5882 a(q d 338)$ phosphorylation-defective mutants. For statistics and additional trials in (a),

589 (b), and (j), see Supplementary Table 1.

590

591

a

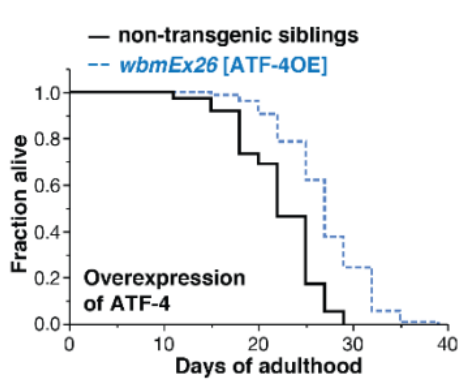

d

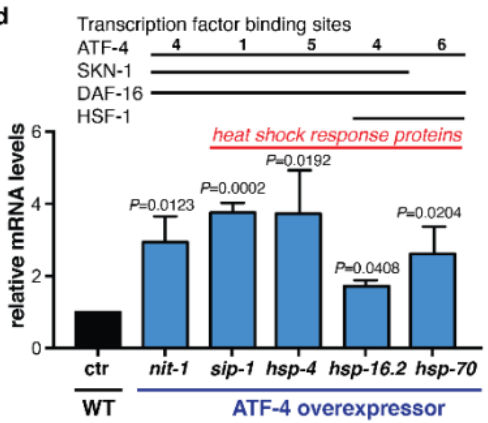

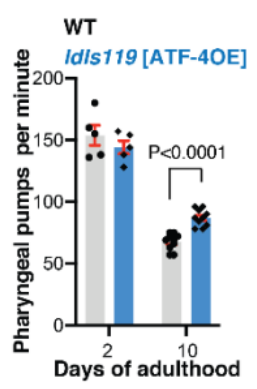

e

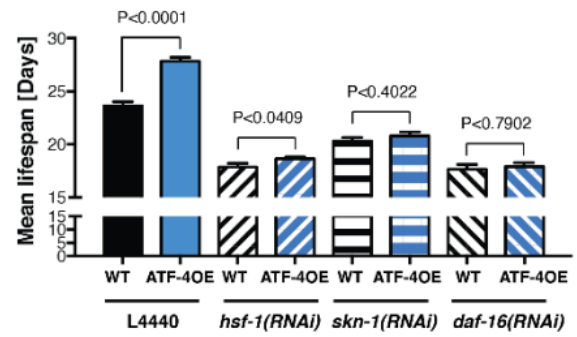

RNA-sequencing
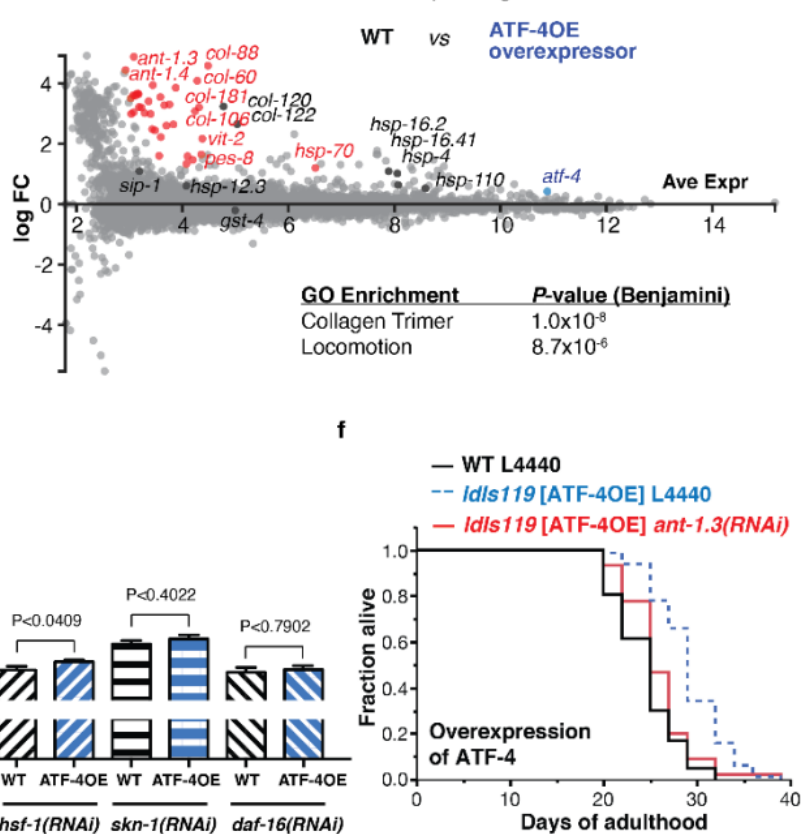

592

Figure 3. ATF-4 overexpression is sufficient to increase lifespan. a Transgenic animals (wbmEx26 [Patf-4::ATF-4(gDNA)::GFP]) that overexpress ATF-4 (ATF-4OE) live longer compared to their non-transgenic siblings. b Pharyngeal pumping rate is similar at day 2 of adulthood between ATF-4OE (Idls119 [Patf-4::ATF-4(gDNA)::GFP]) and WT, but higher in ATF-4OE at day 10 of adulthood, suggesting an improved 
598 healthspan. For the complete time-course of pharyngeal pumping rate during ageing,

599 see Supplementary Table 2. Mean \pm SEM. Unpaired two-tailed $t$-test. c MA (log ratio

600 and mean average)-plot of RNA sequencing analysis comparing ATF-4OE(Idls119) to

601 abs log FC relative to WT. In red, highlighted genes with FDR $<0.1$ and $\log \mathrm{FC}>1$

602 compared to WT. In black, genes with FDR $>0.1$. Details are in Supplementary Table

603 3. d Validation by qRT-PCR of genes differentially expressed in ATF-4OE(Idls119),

604 using two new independent biological samples of over 200 animals each. Mean \pm

605 SEM. $P$ values relative to WT determined by one sample $t$-test, two-tailed, hypothetical

606 mean of 1 . The numbers of ATF4 binding sequences (-TGATG-) $)^{27,28}$ are indicated in

607 Supplementary Table 4. The DAF-16 and SKN-1 transcription factor binding sites are

608 based on chromatin immunoprecipitation ChIP data from www.modencode.org

609 (Supplementary Table 5). e Longevity conferred by ATF-4OE(Idls119) is abolished by

610 knockdown of $h s f-1, s k n-1$, or daf-16. Mean \pm SEM. f The mitochondrial ATP

611 translocase ant-1.3 is required for ATF-4 overexpression-induced longevity. For

612 statistical details and additional lifespan trials in (a), (e), and (f), see Supplementary

613 Table 1.

614 

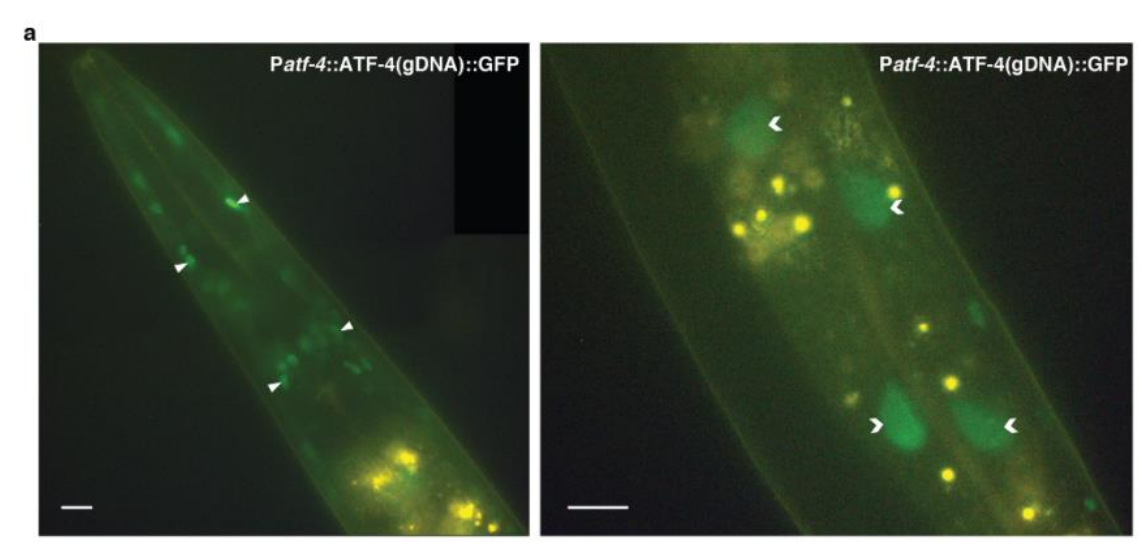

c
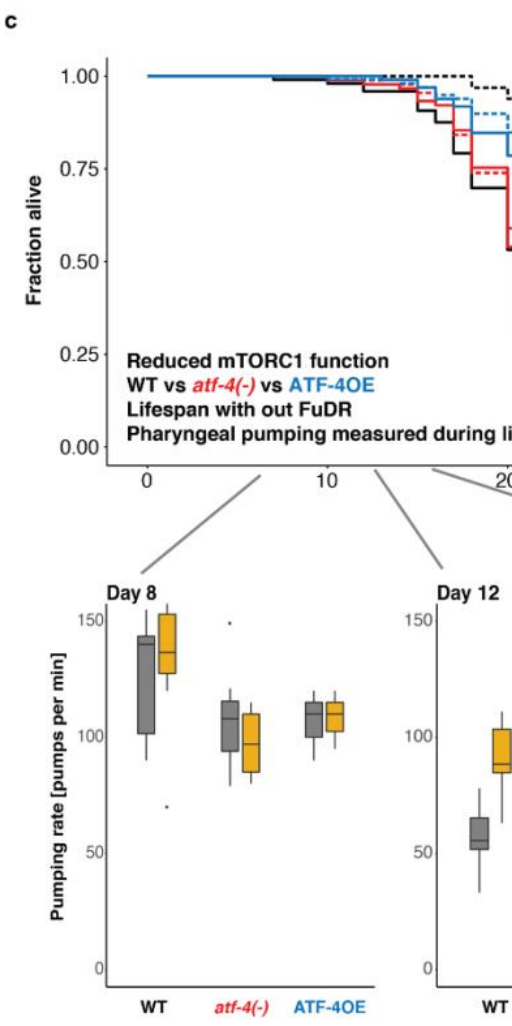

- WT control

-- WT raga-1(RNAi)

- atf-4(tm4397) control

-- atf-4(tm4397) raga-1(RNAi)

- Idls119 ATF-4OE control

-- Idls119 ATF-4OE raga-1(RNAi)

615

616

Extended Data Figure

2. Overexpression of ATF-4

617 Representative images showing the expression of ATF-4 in the head (left) and mid-

618 body (right). ATF-4::GFP (Idls119) is displayed in green and found predominantly in

619 nuclei (nuclei of head neurons or glia indicated by arrowheads, intestinal nuclei

620 indicated by chevrons). Yellow puncta are autofluorescent gut granules. $100 \times$

621 magnification. Scale bar $=10 \mu \mathrm{m}$. b Western blots and quantification showing ATF-

4::GFP levels in day-1-adult transgenic ATF-4OE(Idls119) either treated with DMSO (ctr) or $35 \mu \mathrm{g} / \mathrm{mL}$ tunicamycin for 6 hours. $\mathrm{n}=3$ replicates. Mean \pm SEM. One-sample

$624 t$-test, two-tailed, a hypothetical mean of 1. c Pharyngeal pumping measurements 
625 across the lifespan comparing WT (N2), atf-4(tm4397) mutants, and ATF-

$6264 \mathrm{OE}($ Idls 119) treated with either empty vector control RNAi (L4440) or raga-1(RNAi),

627 on plates that do not contain FUdR. See Supplementary Table 2 for raw data.

628 

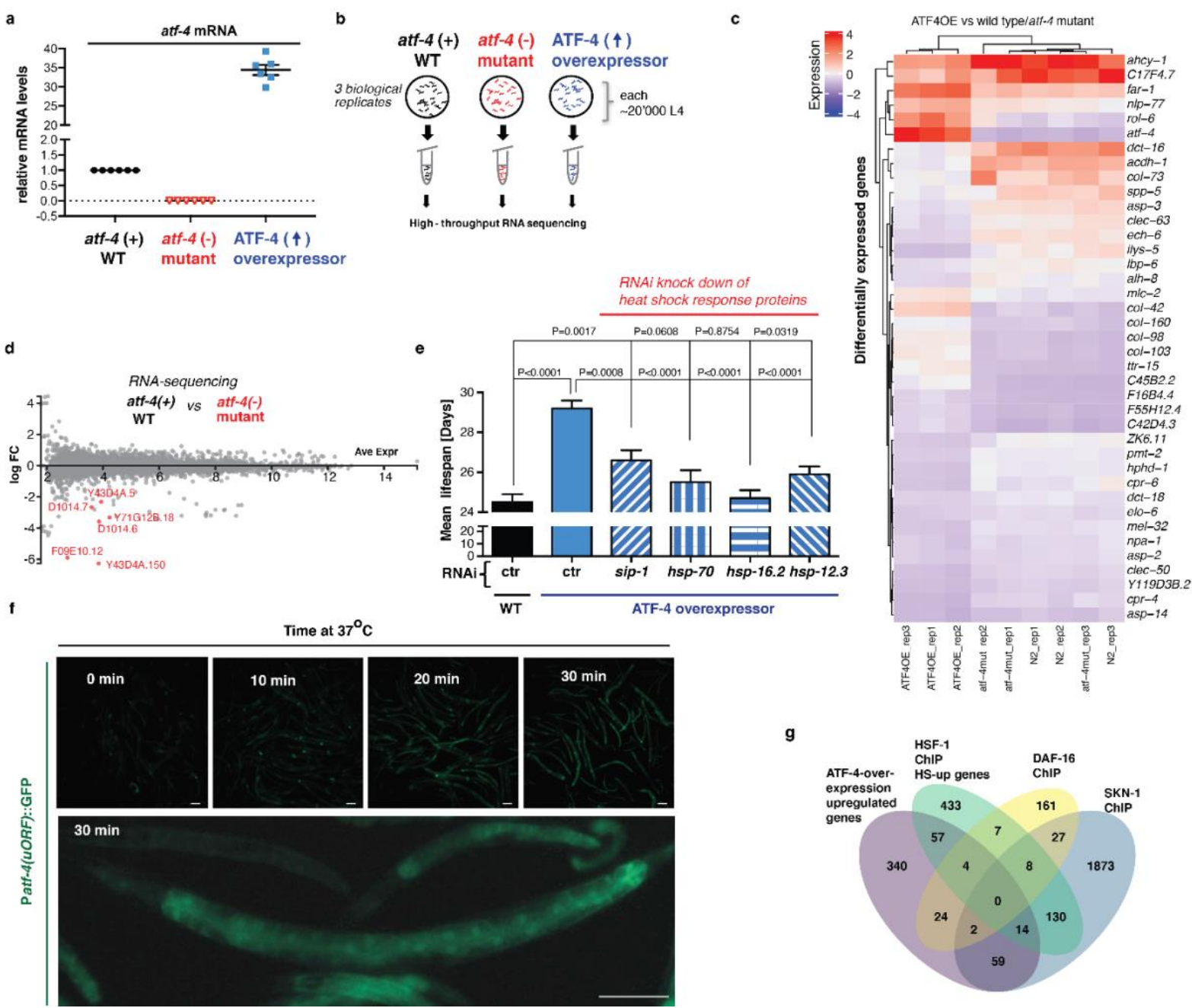

630 Extended Data Figure 3. RNA-sequencing reveals transcriptional targets of ATF-

4. a Quantification of atf-4 mRNA expression levels in atf-4(tm4397) mutants (atf-4(-)) and ATF-4OE $(/ d / s 119)$ relative to wild type (atf-4(+) WT) animals by qRT-PCR. The same samples were used for RNA sequencing. $n=3$ independent biological replicates of about 20, 000 L4 C. elegans. P values for both atf-4(tm4397) or ATF-4OE(Idls119) are $<0.0001$ relative to WT determined by one-sample $t$-test, two-tailed, a hypothetical mean of 1. b Schematic representation of sample collection for RNA sequencing. See

637 Materials and methods for details. c Hierarchical clustering heatmap of the genes that are most differentially regulated in either direction when comparing ATF-4OE $(/ d / s 119)$ to WT and atf-4(tm4397) mutants (atf-4 (-) mutant). As expected, atf-4 is in the top gene set. The collagen rol-6 is the co-injection marker for the transgenic Idls 119 . 
641 Independent biological replicates are indicated as "rep\#". For details and raw data see

642 Supplementary Table 3. d MA (log ratio and mean average)-plot of RNA sequencing

643 analysis comparing atf-4(tm4397) mutants (atf-4 (-) mutant) to absolute log fold-

644 change (FC) relative to WT. In red, highlighted genes with a false discovery rate (FDR)

$645<0.1$ and abs log FC $>1$ to WT. Details in Supplementary Table 3. e Longevity

646 conferred by ATF-4 overexpression (Idls119) is blunted by knockdown of sip-1, hsp-

647 70, $h s p-16.2$, or $h s p-12.3$. Mean \pm SEM. $\mathrm{P}$ values are relative to WT on empty vector

648 RNAi (L4440). For statistical details see Supplementary Table 1. f Representative

649 images that heat increases Patf-4(uORF)::GFP transgene expression. Bottom panel

650 shows higher magnification. Anterior to the right, ventral side down. Scale bar $=100$

$651 \mu \mathrm{m} . \mathbf{g}$ Venn diagram showing the overlap of ATF-4 overexpression-upregulated genes

652 with genes that were bound directly by SKN-1, DAF-16, and HSF-1 in chromatin

653 immunoprecipitation (ChIP) studies. For details and references see Supplementary

654 Table 5.

655 

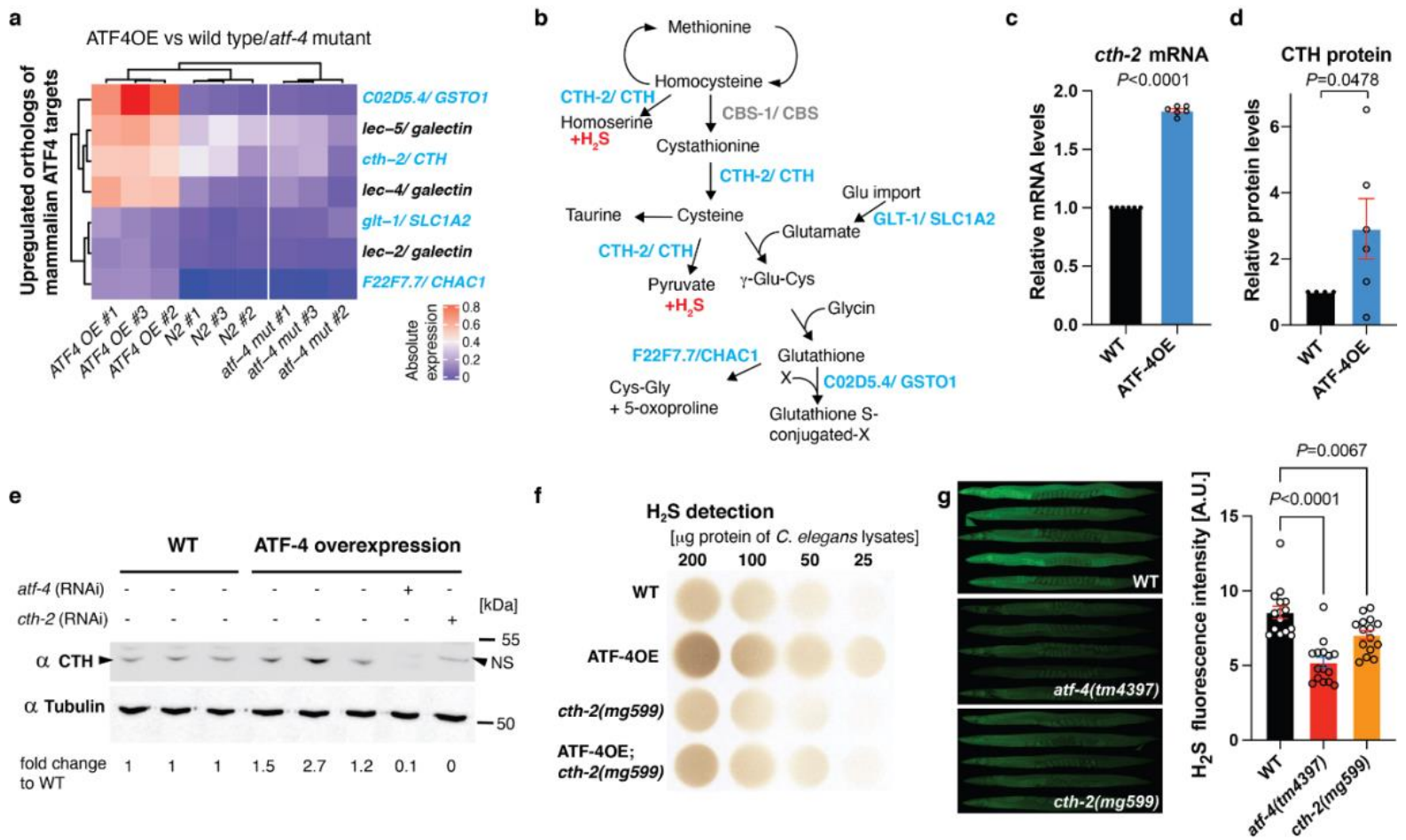

h
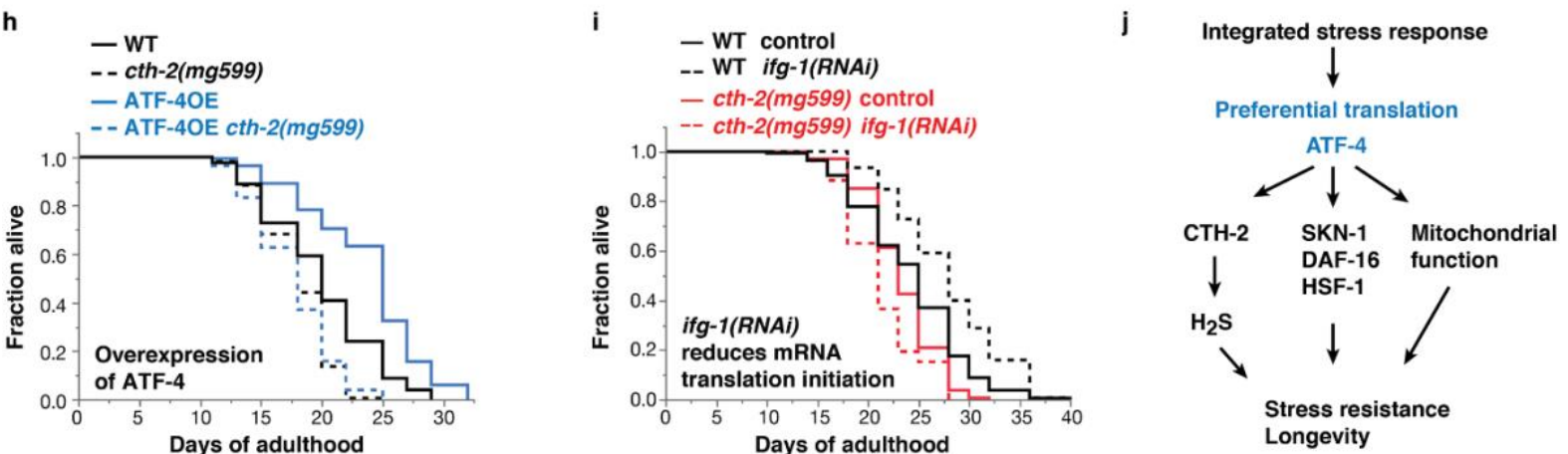

Figure 4. ATF-4 overexpression increases $\mathrm{H}_{2} \mathrm{~S}$ levels via cystathionine gamma-

lyase, which is required for longevity. a Heatmap of gene expression in ATF-4OE

659 (Idls119), wild type (WT), and atf-4(tm4397) showing genes whose orthologs are

660 directly regulated by mammalian ATF4 (Details are in Materials and Methods,

661 Supplementary Table 4). Absolute levels of expression were compared. Genes in light

662 blue are predicted to be involved in the transsulfuration pathway, which is shown in

663 (b). c ATF-4OE(Idls119) showed higher cth-2 mRNA levels compared to WT by qRT-

664 PCR. $n=3$ independent biological samples in duplicates (each over 200 L4 worms).

665 Mean \pm SEM. $P$ values relative to WT determined by one-sample $t$-test, two-tailed, a 666 hypothetical mean of 1. d Quantification of CTH protein levels in ATF-4OE(Id/s119) 
667 compared to WT. $n=6$ independent biological trials probed in 3 western blots. One-

668 tailed $t$-test. e Western blots showing an ATF-4-induced increase in CTH levels was

669 abolished by atf-4 or cth-2 knockdown. NS = non-specific band. $\mathbf{f}$ ATF-4

670 overexpression increases $\mathrm{H}_{2} \mathrm{~S}$ production capacity in a cth-2-dependent manner.

671 Additional biological trials are shown in Extended Data Fig. 4d. For $\mathrm{H}_{2} \mathrm{~S}$ quantification,

672 see Supplementary Table 12. g Representative fluorescent microscopy images and

673 quantification showing that $\mathrm{H}_{2} \mathrm{~S}$ levels in vivo is decreased in either atf-4(tm4397) or

674 cth-2(mg599) mutants compared to WT. Data are represented as mean + SEM. $P$

675 values to WT are unpaired $t$-test, two-tailed. $\mathbf{h}$ Lifespan extension induced by ATF-4

676 overexpression depends upon cth-2. i Lifespan extension induced by ifg-1 knockdown

677 requires cth-2. j Model for how ATF-4 promotes stress resistance and longevity. For

678 statistical details and additional trials in (h) and (i), see Supplementary Table 7.

679 
a

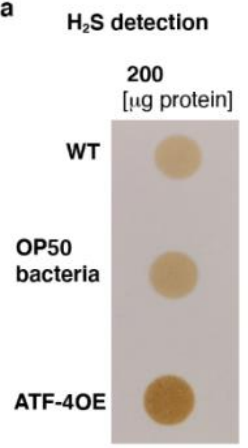

d
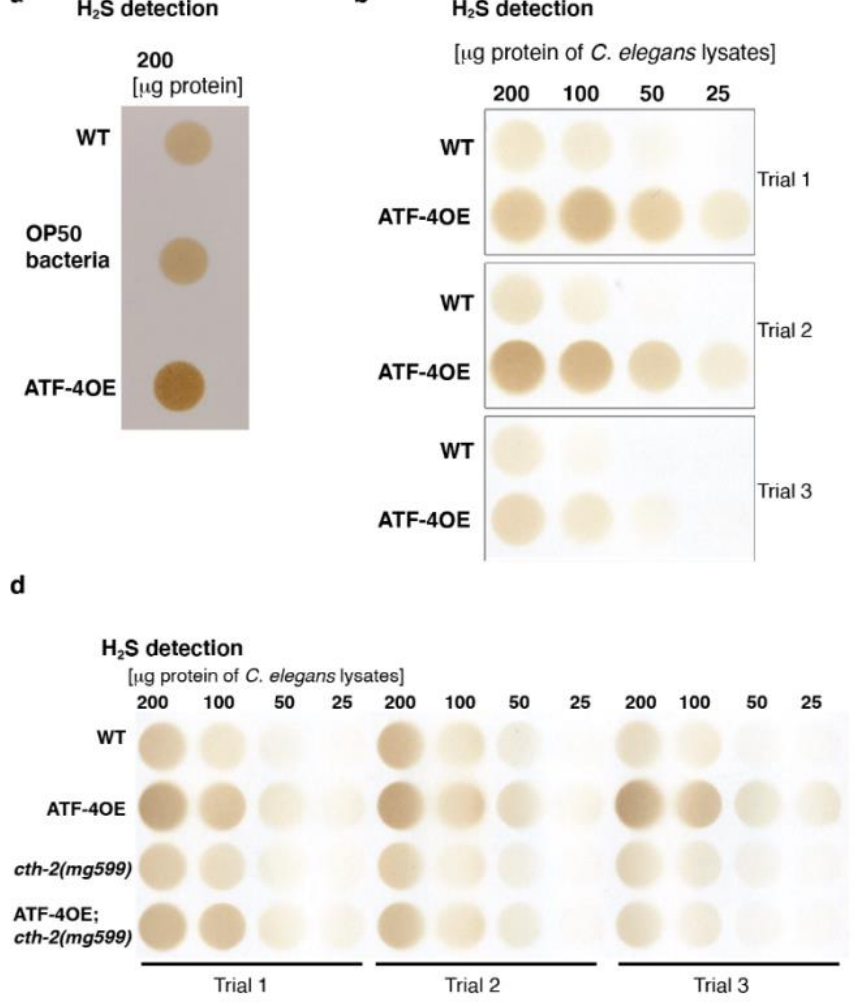

c

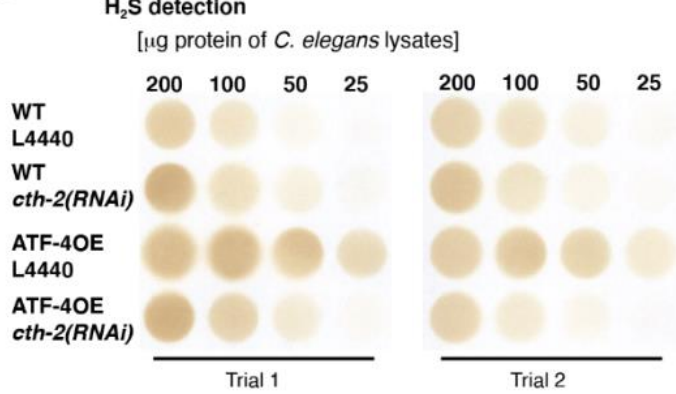

e
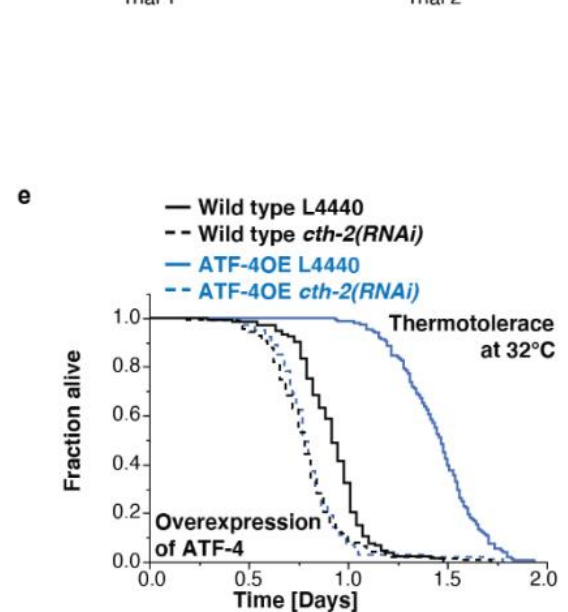

Trial 2

680 


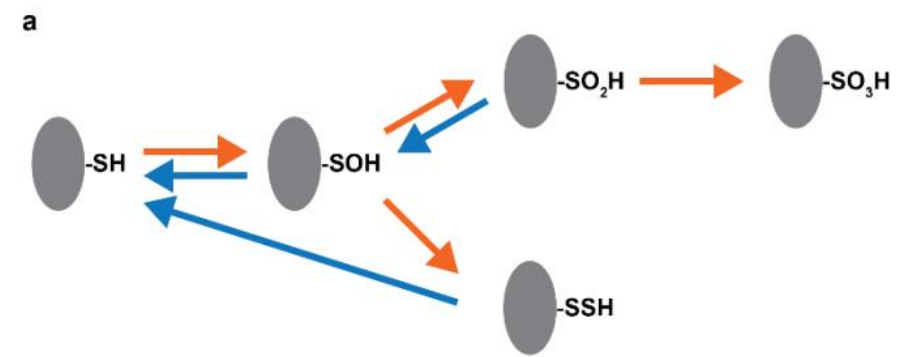

693
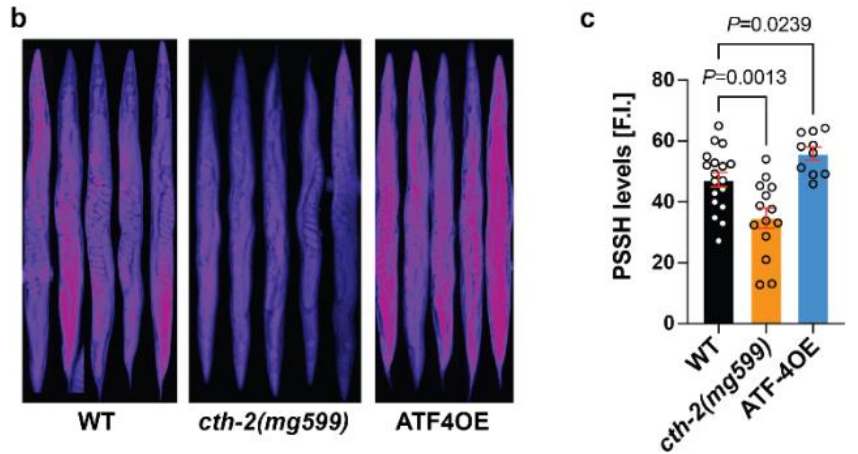

694 Figure 5. ATF-4 and CTH-2 regulate protein persulfidation levels. a Schematic

695 diagram showing that the thiol group $(-\mathrm{SH})$ of reactive cysteine residues in proteins 696 can undertake various redox states. Sulfenylation (-SOH) can be reversed, 697 particularly efficiently through the intermediate of persulfidation (-SSH), but 698 sulfinylation $\left(-\mathrm{SO}_{2} \mathrm{H}\right)$ is reversible only within peroxiredoxins and sulfonylation $\left(-\mathrm{SO}_{3} \mathrm{H}\right)$ 699 is irreversible ${ }^{35,37}$. Arrows in orange indicate oxidation processes while those in blue 700 indicate reduction processes. b Representative fluorescent images and quantification

701 (c) showing that ATF-4OE exhibited higher persulfidation levels, while cth-2(mg599) 702 animals exhibited lower global persulfidation levels, compared to WT. Data are 703 represented as mean + SEM. $P$ values to WT are unpaired $t$-test, two-tailed. 
a

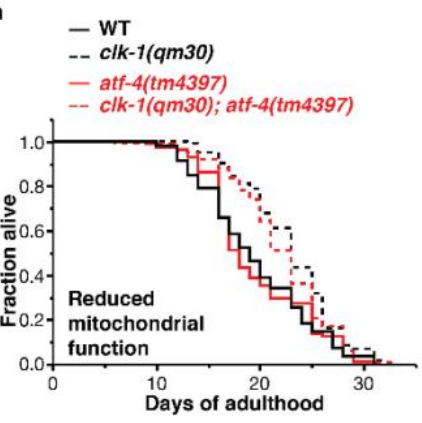

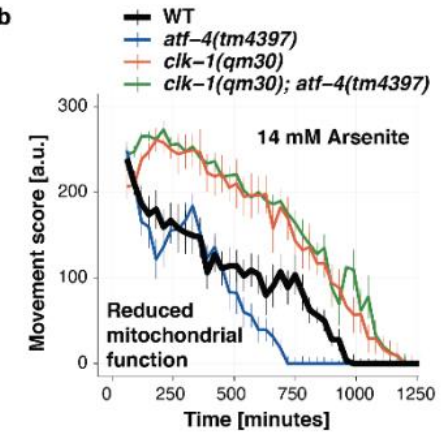

$$
\begin{array}{ll}
-\mathrm{wT} & + \text { raga-1(ok386) } \\
+ \text { atf-4(tm4397) } & \text { - raga-1(ok386); atf-4(tm4397) } \\
& \text { - isp-1(qm150) } \\
& \text { - isp-1(qm150); atf-4(tm4397) }
\end{array}
$$

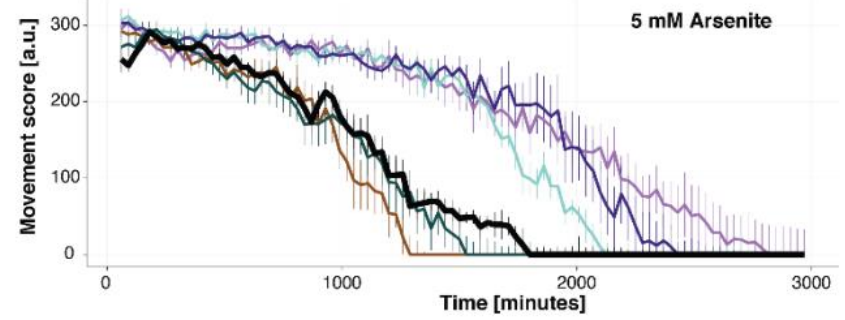

e

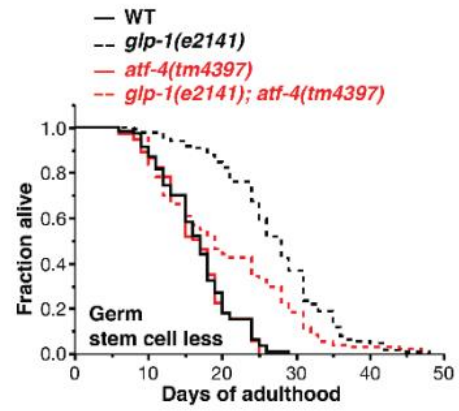

CTH mRNA expression level

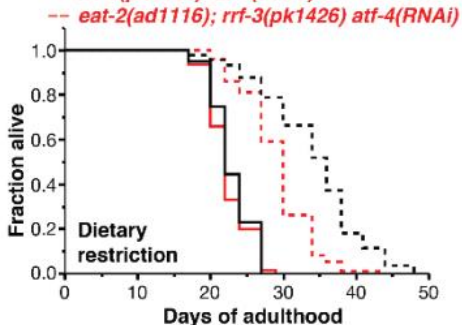

Days of adulthood

707 Extended Data Figure 5. A partial role for ATF-4 in various lifespan extension programs. a The longevity of $c / k-1(q m 30)$ animals (impaired mitochondrial function) 
710 mutants does not require atf-4. c Loss of atf-4 suppresses the oxidative stress

711 resistance of raga-1(ok386) mutants (reduced mTORC1 activity), but not that of

712 reduced mitochondrial function mutant isp-1(qm150). d Longevity arising from

713 reduced insulin/IGF-1 signalling by adult-specific knockdown of the daf-2 receptor is

714 partially suppressed by atf-4(tm4397) mutation. e The atf-4(tm4397) mutation partially

715 suppresses the longevity of glp-1(e2141) mutants (genetic germline stem cell

716 ablation). $f$ Knockdown of atf-4 partially suppresses the longevity of the genetic DR-

717 related model eat-2(ad1116) in the RNAi-sensitized rrf-3(pk1426) background. $\mathbf{g}$ CTH

718 mRNA expression levels in long-lived over control mice, analyzed from publicly

719 available expression datasets (Supplementary Table 11). Data are grouped and

720 colored by interventions and represented as Mean \pm SEM. The meta data of the samples are summarized by colored tiles indicating first the tissue of origin, followed by the sex and then the age group of the mice in each experiment. Animals sacrificed before 16 weeks of age were classified as "young", between 16 to 32 weeks as

724 "middle-aged" and animals above 32 weeks as "old". If no meta information could be 725 found, it was labelled as "not specified". For statistical details and additional trials in (a), (d)-(f), see Supplementary Table 1. For statistical details and additional trials in (b), (c), and see Supplementary Tables 8. 

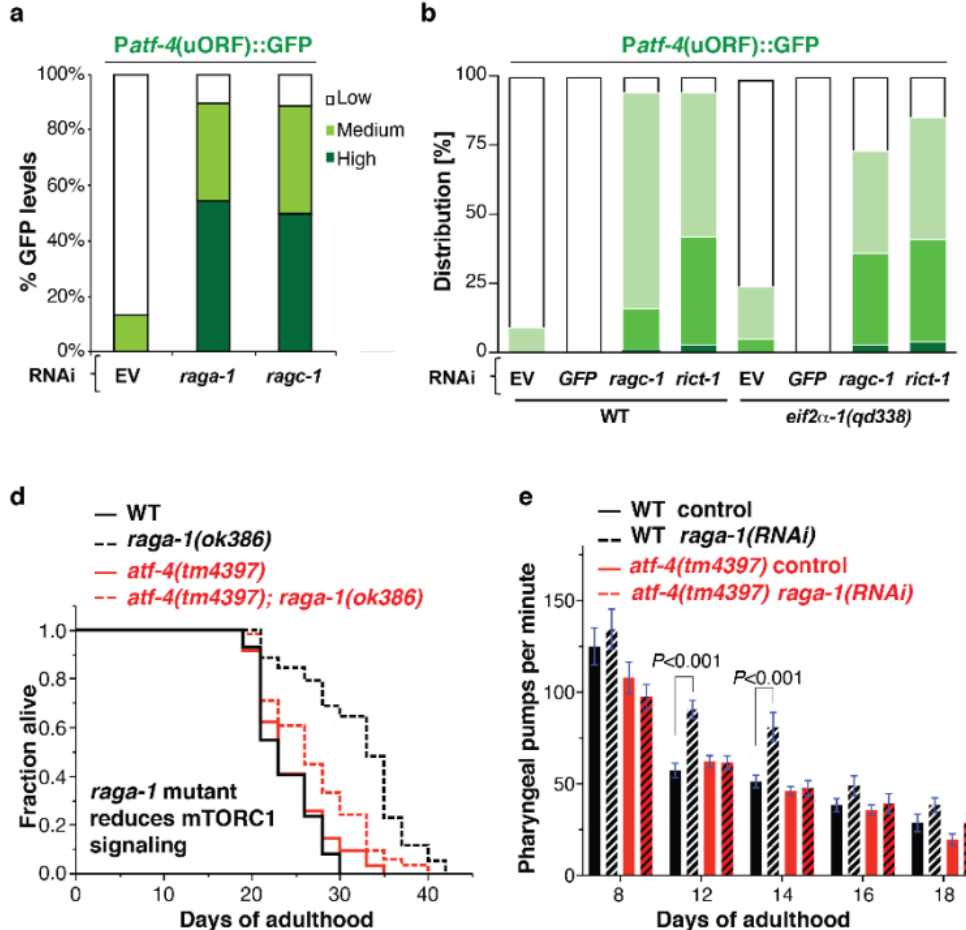

g

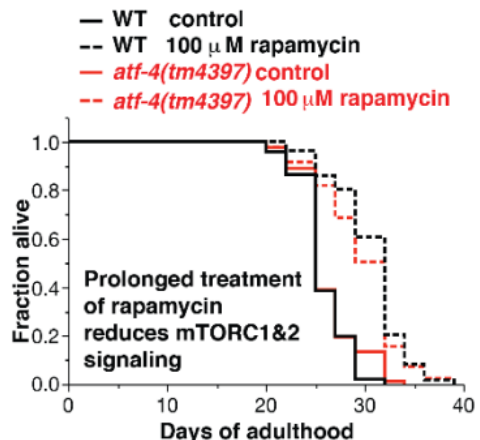

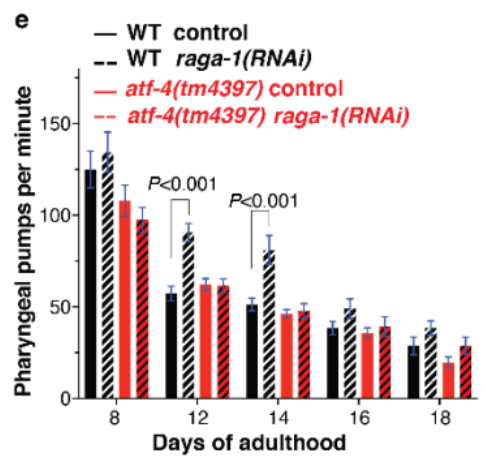

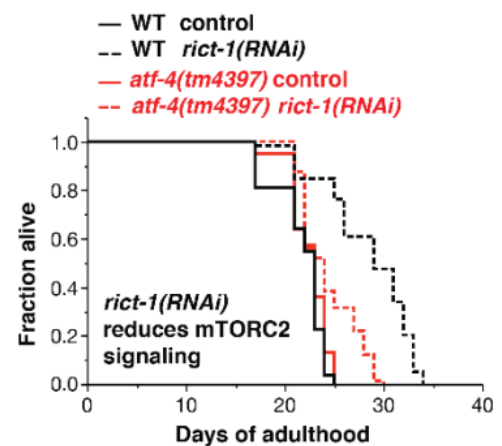

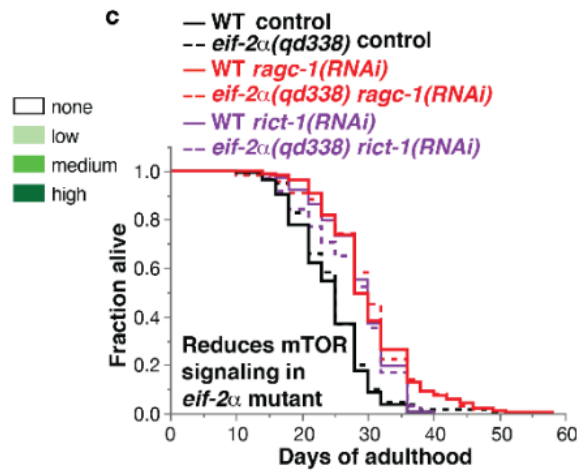

- WT control
-- WT raga-1(RNAi)

- att-4(tm4397) control

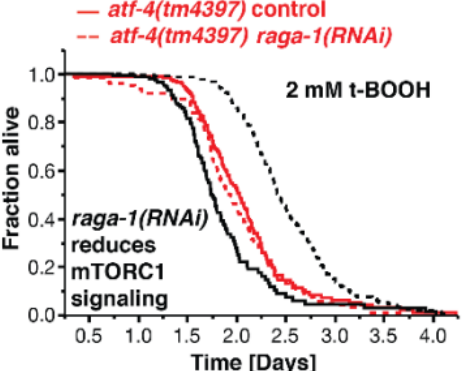

i $\quad-$ WT control

- WT control

-- WT rict-1(RNAi)

-.. WT ragc-1; rict-1(RNAi)

- att-4(tm4397) control

-... atf-4(tm4397) ragc-1(RNAi)

-- atf-4(tm4397) rict-1(RNAi)

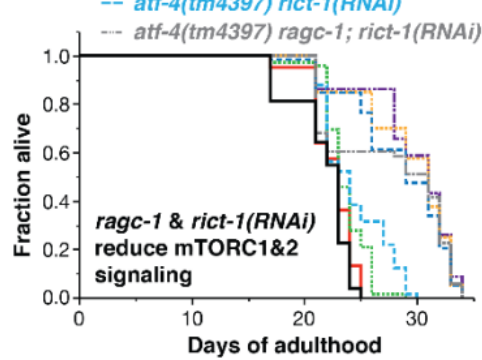

732 Figure 6. ATF-4 is essential for longevity from reduced mTORC1 activity. a

733 Inhibition of $\mathrm{mTORC} 1$ by either raga-1 or ragc-1 knockdown led to preferential

734 translation of ATF-4. RNAi treatments were initiated at the L4 stage, with GFP intensity scored at day 3 of adulthood. $\mathbf{b}$ Inhibition of mTORC1 or mTORC2 by knockdown of ragc-1 or rict-1, respectively, leads to preferential translation of ATF-4. Similar effects were observed in WT and eif2 $\alpha(q d 338)$ mutants. c Post-development knockdown of raga-1 or rict-1 extends lifespan in both WT and eif2 $\alpha(q d 338)$ mutants. d Mutation in raga-1 increases lifespan in an atf-4-dependent manner. e Reducing mTORC1 signalling by adulthood specific raga-1 knockdown improves healthspan dependent 
741 upon atf-4, as assessed by pharyngeal pumping rate. Mean \pm S.E.M. $P$ values relative

742 to WT of the corresponding day with One-way ANOVA with post hoc Dunnett's multiple

743 comparisons test. f Adult-specific knockdown raga-1 increases oxidative stress

744 resistance (2 $\mathrm{mM}$ tert-butyl hydrogen peroxide (tBOOH)) in an atf-4-dependent

745 manner. RNAi was started at the L4 stage, and stress resistance was measured at 746 day 3 of adulthood with the lifespan machine (See Supplementary Table 10 for

747 details). $\mathbf{g}$ Rapamycin treatment during adulthood extends lifespan independently of

748 atf-4. h Adult-specific knockdown of the mTORC2 subunit rict-1 extends lifespan in an

749 atf-4-dependent manner. i Adult-specific inactivation of both mTORC1 and mTORC2

750 increases lifespan independently of atf-4. For statistical details and additional trials in

751 (a), (b), see Supplementary Table 9. For statistical details and additional lifespan trials in (c), (d), (g)-(i), see Supplementary Table 1. 


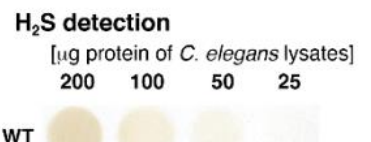

atf-4(tm4397) \begin{tabular}{c|c}
$\bar{\delta}$ & raga-1(ok386) \\
\hline E & $\begin{array}{l}\text { raga-1(ok386); } \\
\text { atf-4(tm4397) }\end{array}$
\end{tabular}

c

- WT control

-- WT cth-2(RNAi)

- raga-1(ok386) control

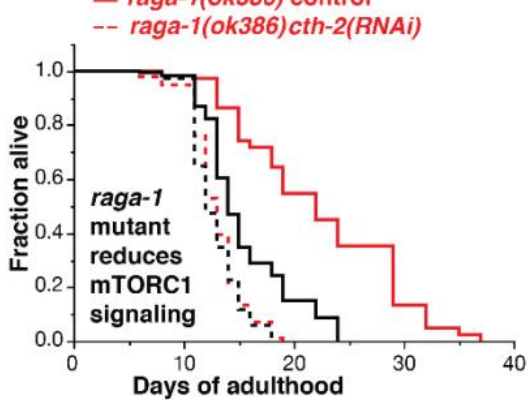

e
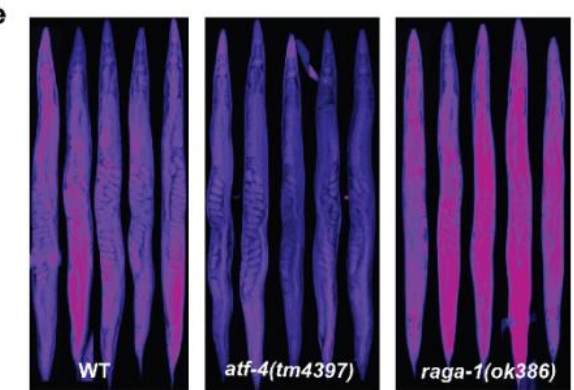

g

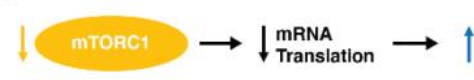

b

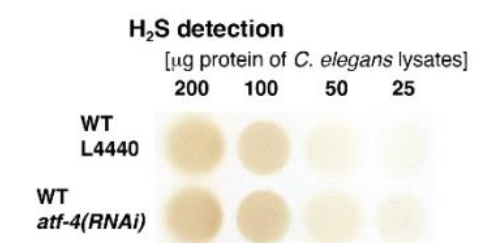

d $\quad$ - WT control

- WT ragc-1(RNAi)

-- cth-2(mg599) ragc-1(RNAi)

- WT rict-1(RNAi)

-- cth-2(mg599) rict-1(RNAi)

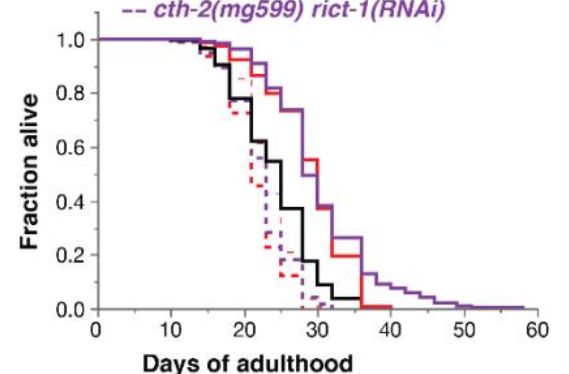

Days of adulthood

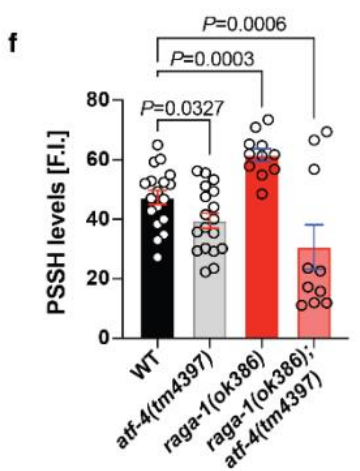

Figure 7. Longevity from mTOR inhibition upregulates $\mathrm{H}_{2} \mathrm{~S}$ and requires cth-2.

a Assay of $C$. elegans lysates showing that raga-1 mutation increased $\mathrm{H}_{2} \mathrm{~S}$ production capacity in an atf-4-dependent manner. Two additional independent biological trials are in Extended Data Fig. 6d. b Assay of C. elegans lysates showing that rict-1 mutation increased $\mathrm{H}_{2} \mathrm{~S}$ production capacity in an atf-4-dependent manner. An additional independent biological trial is in Extended Data Fig. 6e. c Longevity of raga-

761 1(ok386) mutants is ablated by cth-2 knockdown. This particular experiment was performed at $25^{\circ} \mathrm{C}$. d Longevity induced by adult-specific knockdown of either raga-1 
763 or rict-1 depends upon cth-2. e and f Representative images showing persulfidation

764 levels in WT (N2), cth-2 (mg599), atf-4 (tm4397), raga-1 (ok386), and raga-1;atf-4

765 mutants. Data are represented as mean + SEM. $P$ values to WT are unpaired $t$-test,

766 two-tailed. $\mathbf{g}$ Inhibition of mTORC1 promotes longevity by increasing ATF-4

767 expression and stimulating $\mathrm{H}_{2} \mathrm{~S}$ production. For $\mathrm{H}_{2} \mathrm{~S}$ quantification in (a) and (b), see

768 Supplementary Table 12. For statistical details and additional lifespan trials in (c) and

769 (d), see Supplementary Table 1.

770 

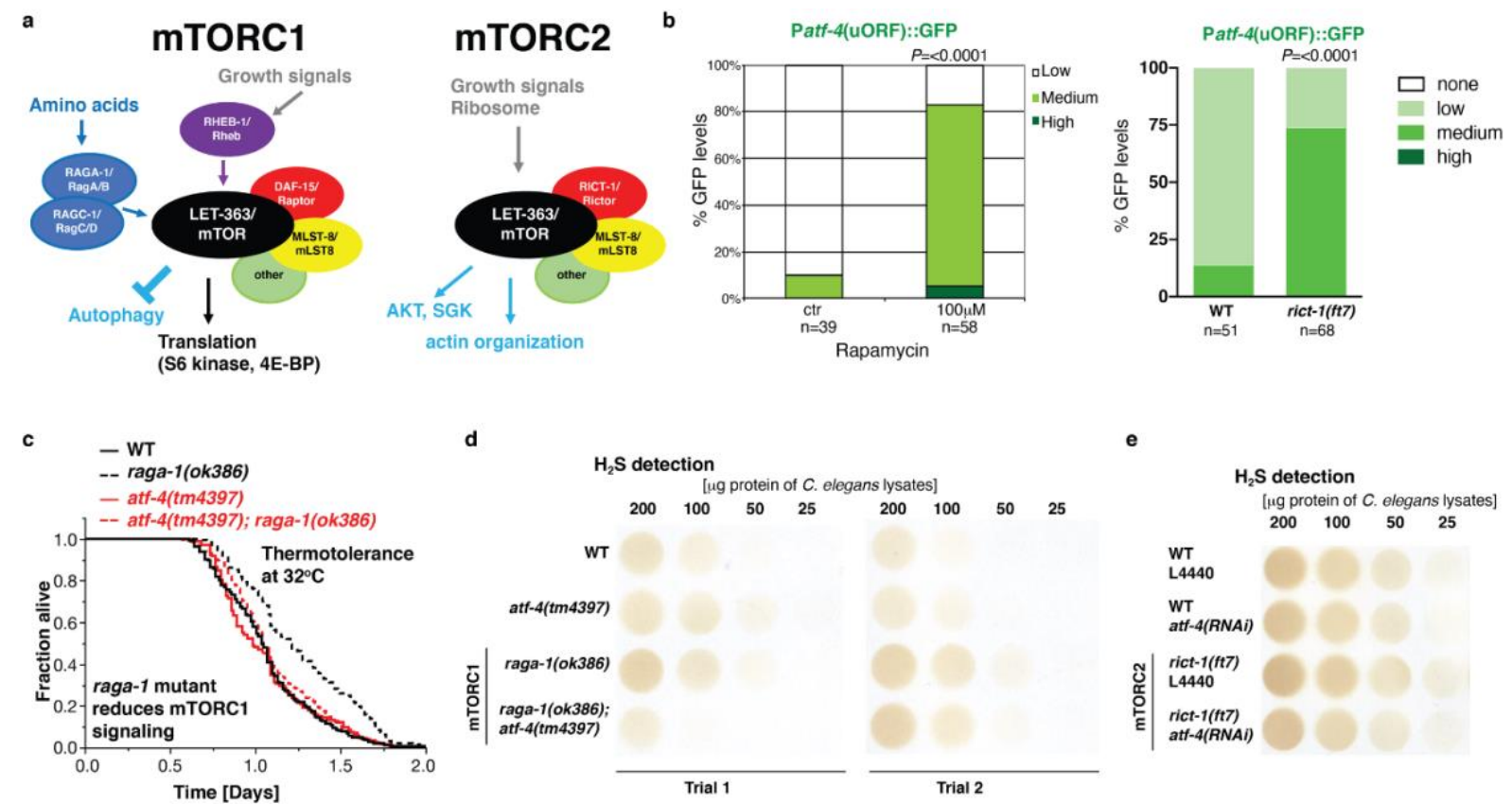

d
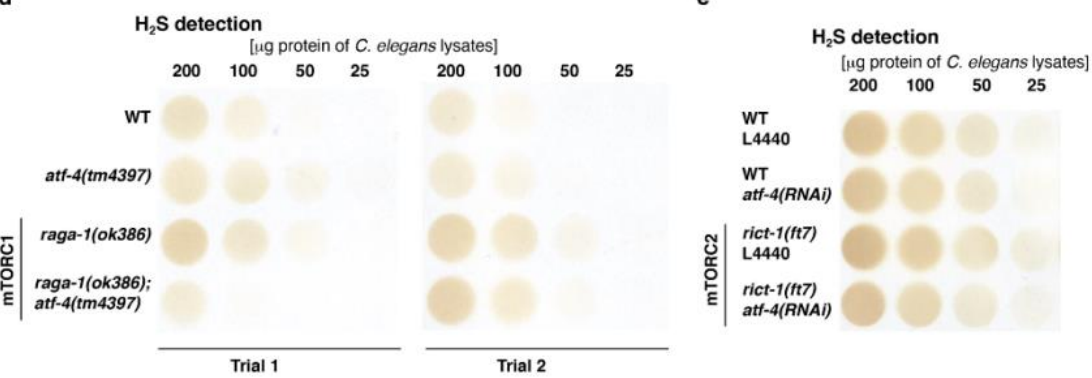

f

- WT control

- raga-1(ok386) control

-- raga-1(ok386)cth-2(RNAi)
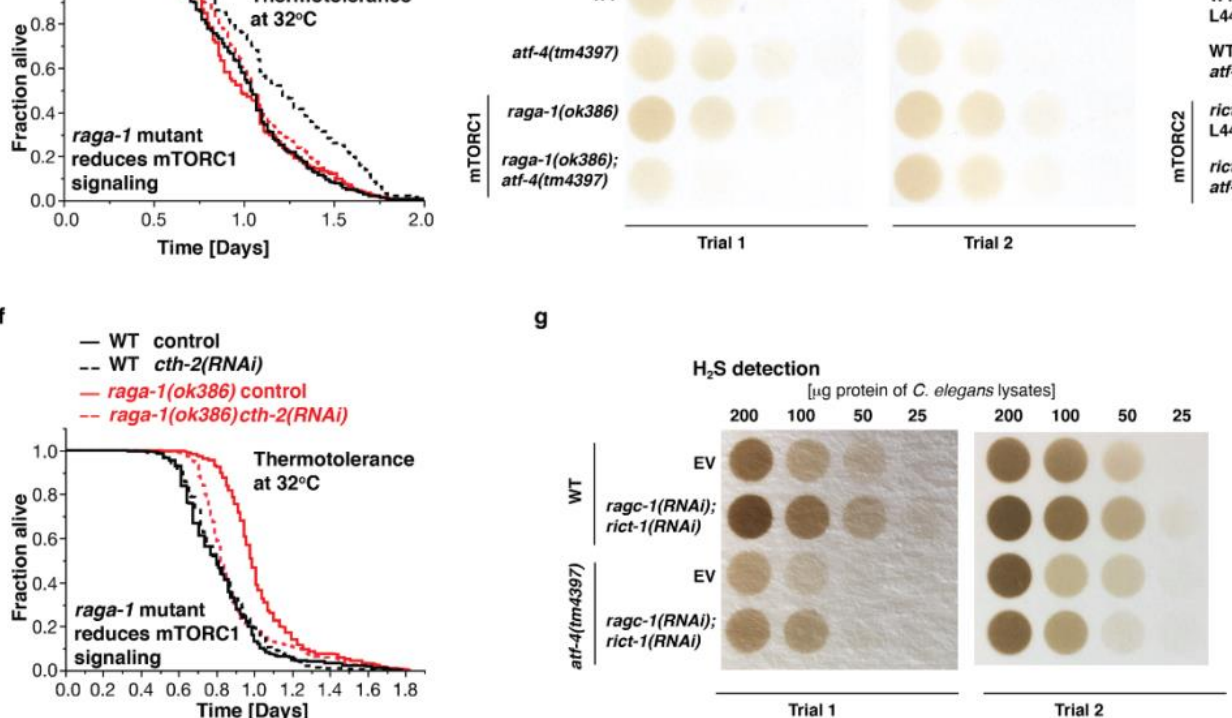

771

Extended Data Figure 6. Preferential atf-4 translation and $\mathrm{H}_{2} \mathrm{~S}$ signalling upon

reduced mTOR signalling. a Schematic diagram showing the composition, regulation, and functions of the two mTOR complexes (mTORC1 and mTORC2) adapted from ${ }^{6}$. b Rapamycin treatment (left) or rict-1(ft7) mutation (right) leads to preferential translation of ATF-4. Rapamycin treatment was initiated at L4. GFP intensity was scored at day 3 of adulthood. $P$ values were determined by $\mathrm{Chi}^{2}$ test. c Increased heat stress resistance $\left(32^{\circ} \mathrm{C}\right)$ of raga-1(ok386) mutants depends on atf-4. d Two additional independent biological trials of Fig. 7a. e One additional independent

780 biological trial of Fig. $7 \mathrm{~b}$. $\mathrm{f}$ Increased heat stress resistance $\left(32^{\circ} \mathrm{C}\right)$ of raga-1(ok386) mutants depends on cth-2. $\mathbf{g ~ H}_{2} \mathrm{~S}$ production capacity assay from $C$. elegans lysates showing that simultaneous knockdown of both raga-1 and rict-1 increases $\mathrm{H}_{2} \mathrm{~S}$ 
783 production capacity in an atf-4-independent manner. For statistical details and

784 additional trials in (c) and (f), see Supplementary Table 7; Quantification of $\mathrm{H}_{2} \mathrm{~S}$ assays

785 in $(\mathbf{d}),(\mathbf{e})$, and $(\mathbf{g})$ are in Supplementary Table 12.

786 
Materials and Methods

788

\section{Strains}

789 Caenorhabditis elegans strains were maintained on NGM plates and OP50

790 Escherichia coli bacteria. LD1499 [Patf-4(uORF)::GFP::unc-54(3'UTR)] was made by

791 Chi Yun (1.8kb promoter 5' of atf-4 including both uORFs into pPD95.75, personal 792 communication with Chi Yun and David Ron) ${ }^{57}$. All strains used are listed in 793 Supplementary Table 13.

794

\section{Generation of transgenic lines}

796 Construction of a translational fusion of ATF-4 with GFP. The plasmid pWM48 (Patf797 4::ATF-4(gDNA)::GFP::unc-54(3'UTR)) was generated by introducing the $1.8 \mathrm{~kb}$ promoter region 5 ' of atf-4 and the atf-4 genomic sequence into pAD1. This construct was used to generate two independent transgenic lines: wbmEx26 [pWM48 (Patf4::ATF-4(gDNA)::GFP::unc-54(3'UTR), pRF4 (rol-6(su1006))] and wbmEx27 [pWM48 (Patf-4::ATF-4(gDNA)::GFP::unc-54(3'UTR), pRF4 (rol-6(su1006))]. UV irradiation was used for integration resulting in Idls119 from wbmEx26 and Idls120-1 from wbmEx27, which were outcrossed 8-10x against N2.

804

\section{Genomic organisation and alignments}

806 Alignment of C. elegans ATF-4 (T04C10.4, WBGene00000221, 208 amino acids, 807 www.wormbase.org) with human ATF4 (350 amino acids, P18848; www.uniprot.org) 808 or ATF5 (282 amino acids, Q9Y2D1; www.uniprot.org) sequences was performed by

809 T-COFFEE (Version_11.00.d625267). The atf-4 genomic representation was made

810 using Exon-Intron Graphic Maker (http://wormweb.org/exonintron) from Nikhil Bhatla.

811 DNA and mRNA sequences were from www.wormbase.org (WS258). For human 
812 ATF4 GenBank BC008090 mRNA sequence was used. The uORFs were predicted

813 with ApE- A plasmid Editor v2.0.50b3. For amino acid alignments T-COFFEE

814 (Version_11.00.d625267) was used.

815

816 Ribosome profiling analysis

817 Ribosome profiling sequencing data were downloaded from the NCBI Sequence Read

818 Archive (S.R.A.) (http://www.ncbi.nlm.nih.gov/sra/) under accession number

819 SRA055804. Data were analyzed as described ${ }^{54}$ : Data analysis was performed with

820 the help of Unix-based software tools. First, the quality of raw sequencing reads was

821 determined by FastQC (Andrews, S. FastQC (Babraham Bioinformatics, 2010)).

822 Reads were then filtered according to quality via FASTQ for a mean PHRED quality 823 score above 30 (http://usegalaxy.org/u/dan/p/fastq). Filtered reads were mapped to

824 the worm reference genome (Wormbase WS275) using B.W.A. (version 0.7.5), and

825 S.A.M. files were converted into B.A.M. files by SAMtools (version 0.1.19). Read

826 counts were then assigned to each gene or non-coding RNA. Library sizes were

827 normalized using the EdgeR software package and the TMM normalization mode.

828 Ribosome profiling was computed separately for each library as the average

829 normalized read counts across all for normalized libraries. Thus, the mapped reads

830 were determined and normalized based on library size for each transcript in each

831 ribosome profiling library. The ATF-4 coverage data for each larval stage (L1, L2 and

832 L4 ) and for the whole transcript (including 5'UTR, exons and 3'UTR) were calculated 833 and exported by SAMtools. The stage-specific averaged coverage data for each gene 834 were plotted using R (https://www.r-project.org). 
837 RNAi clones were obtained from the Vidal and Ahringer RNAi libraries. RNAi bacteria

838 cultures were grown overnight in LB with carbenicillin [100 $\mu \mathrm{g} / \mathrm{ml}]$ and tetracycline

$839[12.5 \mu \mathrm{g} / \mathrm{ml}]$, diluted to an OD600 of 1, and induced with $1 \mathrm{mM}$ IPTG and spread onto

840 NGM plates containing tetracycline $[12.5 \mu \mathrm{g} / \mathrm{ml}]$ and ampicillin $[50 \mu \mathrm{g} / \mathrm{ml}]$. For empty

841 RNAi vector (EV) plasmid pL4440 was used as control.

842

843 Manual lifespan assays

844 Adult lifespan was determined either with or without FUdR as described in Ewald and colleagues $^{58}$. About 100 L4 C. elegans per strain were picked onto NGM plates

846 containing OP50 bacteria. The next day, C. elegans (day-1-adults) were transferred

847 onto either NGM plates containing $400 \mu \mathrm{M}$ FUdR and OP50 bacteria or RNAi bacteria.

848 For cycloheximide-treatment lifespan, day-1-adults were transferred on NGM OP50 849 plates either containing the solvent $0.25 \%$ dimethyl sulfoxide (DMSO) alone as a 850 control or cycloheximide (Sigma \#C7698) dissolved in 0.25\% DMSO. The rapamycin 851 lifespan and liquid DR lifespan assays were performed as described ${ }^{6,58}$. Animals were 852 classified as dead if they failed to respond to prodding. Exploded, bagged, burrowed, 853 or animals that left the agar were excluded from the statistics. The estimates of survival 854 functions were calculated using the product-limit (Kaplan-Meier) method. The log-rank 855 (Mantel-Cox) method was used to test the null hypothesis and calculate $P$ values (JMP 856 software v.9.0.2.).

\section{Pharyngeal Pumping}

859 Pharyngeal pumping was assessed as described previously ${ }^{26}$. Pharyngeal pumping 860 was determined by counting grinder movements in 45 second intervals while the 861 animals were feeding on the bacterial lawn. 


\section{Puromycin assay}

864 Puromycin incorporation and detection assays were adapted from previous

865 studies ${ }^{15,59}$. Approximately 500 L4 animals were resuspended in M9 and transferred 866 to NGM plates containing $50 \mu \mathrm{M}$ FUdR seeded with RNAi bacteria clones. After 3 days, 867 worms were collected in M9 and then transferred to S-basal medium. Worms were 868 incubated with $4 \mathrm{ml} \mathrm{S-Basal} \mathrm{that} \mathrm{contained} \mathrm{OP50} \mathrm{and} 0.5 \mathrm{mg} / \mathrm{ml}$ puromycin for $1 \mathrm{hr}$. 869 Afterwards, worms were washed with S-basal for three times. Protein extraction and 870 Western blots for puromycin detection were performed as described below.

\section{Quantitative Real-Time Polymerase Chain Reaction (qRT-PCR) Assays}

873 RNA was isolated with Trizol (TRI REAGENT Sigma), DNAse-treated, and cleaned

874 over a column (RNA Clean \& Concentrator ${ }^{\text {TM }}$ ZYMO Research). First-strand cDNA 875 was synthesized in duplicate from each sample (Invitogen SuperScript III). SYBR 876 green was used to perform qRT-PCR (ABI 7900). For each primer set, a standard curve from genomic DNA accompanied the duplicate cDNA samples. mRNA levels relative to WT control were determined by normalizing to the number of $C$. elegans and the geometric mean of three reference genes (cdc-42, pmp-3, and Y45F10D.4).

880 At least two independent biological replicates were examined for each sample. For 881 statistical analysis, one-sample $t$-test, two-tailed, a hypothetical mean of 1 was used 882 for comparison using Prism 6.0 software (GraphPad).

\section{RNA sequencing}

885 Three independent biological replicates were prepared by using sodium hypochlorite 886 to harvest eggs and overnight L1 arrest in M9 buffer with $10 \mu \mathrm{g} / \mathrm{ml}$ cholesterol to 
synchronize C. elegans. For each sample, about 20000 C. elegans per strain were allowed to develop to the L4 stage under normal growth conditions on NGM OP50 plates at $20^{\circ} \mathrm{C}$ (about 1000 C. elegans per one $10 \mathrm{~cm}$ NGM OP50 plate). WT, atf4(tm4397), and Idls119 were grown at the same time for each biological replicate. $C$. elegans were washed from the culturing NGM plates and washed additional 3 times with M9 buffer to wash away the OP50 bacteria. RNA was isolated with Trizol (TRI REAGENT Sigma), DNAse-treated, and cleaned over a column (RNA Clean \& Center core for sequencing (http://mbcf.dfci.harvard.edu). The RNA Integrity Number (RIN) was then assessed by using the Bioanalyzer 2100 (Agilent Technologies), and only samples with a high RIN score were used to prepare cDNA libraries. All nine samples were multiplexed in a single lane. Single read 50 bp RNA-sequencing with poly $(A)$ enrichment was performed using a HiSeq 2000 (Illumina). We aligned the FASTQ output files to the C. elegans WBcel235 reference genome using STAR 2.4.0j software (http://code.google.com/p/rna-star/) with an average >80\% coverage mapping the reads to the genome. The differential gene expression analysis was performed using Bioconductor (http://bioconductor.org) as described in ${ }^{60}$. Rsubread output files. Transcripts with $<1$ count per million reads were discarded. Counts were

906 scaled to Reads Per Kilobase of transcript per Million mapped reads (RPKM) and 907 deposited as a final output file in (Supplementary Table 3). To analyze the differential expressed genes, we compared atf-4(tm4397), and Idls119 to wild type using Degust

909 (http://degust.erc.monash.edu) with the following settings: RPKM with minimum 5 910 counts using edgeR with a false discovery rate (FDR) of 0.1 and an absolute log fold 911 change (FC) of 1 relative to WT. Results are displayed in MA-plots. Functional 
912 annotation clustering was performed with DAVID using high classification stringencies

913 (https://david.ncifcrf.gov).

914

\section{Comparison of RNA sequencing data with mammalian ATF4 target genes}

916 The RNA-sequencing data described in the previous section was subjected to

917 differential expression analysis using the limma package (Smyth, Gordon K. "Limma:

918 linear models for microarray data." Bioinformatics and computational biology solutions

919 using R and Bioconductor. Springer, New York, NY, 2005. 397-420) available in the

920 programming language $\mathrm{R}$ (Team, R. Core. "R: A language and environment for

921 statistical computing." (2013): 201). The 200 most-upregulated genes that were

922 identified by comparison of ATF4 OE to WT and passed a Benjamini-Hochberg

923 adjusted $P$-value threshold of 0.1 were analyzed further. Mammalian ATF4-specific

924 gene targets were obtained from Quiros et al. $2017^{27}$ and subjected to Ortholist2 to

925 infer $C$. elegans orthologs based on a comparative genomic meta-analysis ${ }^{61}$. The

926 intersection of the most-upregulated genes in our ATF4OE to WT expression analysis

927 and the orthologs of the mammalian ATF4 targets is depicted as a heatmap showing

928 all biological replicates

929 (http://www.bioconductor.org/packages/devel/bioc/html/ComplexHeatmap.html). The

930 atf-4 mutant samples are shown separately since the displayed genes were selected

931 based on the comparison between ATF4OE and WT. The absolute expression levels

932 are displayed in a blue (low) to white (medium) to red (high) color gradient, with genes

933 indicated as gene names or sequence names if the former is not available.

934 Hierarchical clustering was applied to both genes (rows) and samples (columns).

935 Additional information: GO term enrichment yielded a significant $(P=0.047$, Benjamini-

936 Hochberg corrected) enrichment of the membrane raft compartment (lec-2, lec-4, lec- 
937 5) while no significant enrichment for GO biological process, GO molecular function,

938 KEGG- or REACTOME pathways were found.

939

\section{Analysis of CTH expression levels in mice}

941 Publicly-available expression datasets were analyzed to quantify the change of $\mathrm{CTH}$

942 expression levels in long-lived compared to normal-lived mice. A selected subset of

943 comparisons displaying CTH upregulation in longevity is depicted in Fig. 6b, while the

944 full table is provided in Supplementary Table 11. Microarray datasets and platform

945 information were obtained from GEO (https://www.ncbi.nlm.nih.gov/geo/) followed by

946 mapping probes to their corresponding genes and sequencing information was

947 obtained from SRA (https://www.ncbi.nlm.nih.gov/sra) and processed using Trim

948 Galore (https://www.bioinformatics.babraham.ac.uk/projects/trim_galore/) and

949 Salmon ${ }^{62}$. Datasets were centered and scaled, and subsequently, the mean fold

950 change, as well as its standard error, were computed for the CTH gene.

951

952 Manual thermotolerance assays

953 Day-1-adults were placed on NGM OP50 plates (maximum 20 C. elegans per plate)

954 and placed in an incubator at $35^{\circ} \mathrm{C}$. Survival was scored every hour. Animals were 955 classified as dead if they failed to respond to prodding. Exploded animals or animals 956 that moved up on the side of the plate were censored from the analysis. The estimates 957 of survival functions were calculated using the product-limit (Kaplan-Meier) method.

958 The log-rank (Mantel-Cox) method was used to test the null hypothesis and calculate $959 \quad P$ values (JMP software v.9.0.2.).

960

961 Automated survival assays using the lifespan machine 
962 Automated survival analysis was conducted using the lifespan machine described by

963 Stroustrup and colleagues ${ }^{63}$. Approximately 500 L4 animals were resuspended in M9

964 and transferred to NGM plates containing $50 \mu \mathrm{M}$ FUdR seeded with OP50 bacteria,

965 RNAi bacteria supplemented with $100 \mu \mathrm{g} / \mathrm{ml}$ carbenicillin, heat-killed OP50 bacteria,

966 or UV-inactivated E. coli strain NEC937 B (OP50 $\Delta$ uvrA; KanR) containing $100 \mu \mathrm{g} / \mathrm{ml}$

967 carbenicillin. For oxidative stress assays, tBOOH was added to $2 \mathrm{mM}$ to the NGM

968 immediately before pouring and seeding with heat-killed OP50 bacteria. Animals were

969 kept at $20^{\circ} \mathrm{C}$ until measurement. Heat and oxidative stress experiments were

970 performed using regular petri dishes sealed with parafilm, while tight-fitting petri dishes

971 (BD Falcon Petri Dishes, 50x9mm) were used for lifespan experiments. Tight-fitting

972 plates were dried without lids in a laminar flow hood for 40 minutes before starting the

973 experiment. Air-cooled Epson V800 scanners were utilized for all experiments

974 operating at a scanning frequency of one scan per 10 - 30 minutes. Temperature

975 probes (Thermoworks, Utah, U.S.) were used to monitor the temperature on the

976 scanner flatbed and maintain $20^{\circ} \mathrm{C}$ constantly. Animals which left the imaging area

977 during the experiment were censored.

978 Population survival was determined using the statistical software $\mathrm{R}^{64}$ with the survival 979 and survminer (https://rpkgs.datanovia.com/survminer/) packages. Lifespans were 980 calculated from the L4 stage (= day 0 ). For stress survival assays the moment of 981 exposure was utilized to define the time point zero of each experiment.

982

983 Manual oxidative stress assay (arsenite and tBOOH)

984 The manual oxidative stress assays were performed as described in detail in the bio985 protocol $^{65}$. L4 worms were manually picked onto fresh OP50 plates. The next day, $10-$ 98612 day-one old $C$. elegans were transferred into 24 well plates containing $1 \mathrm{~mL}$ M9 
987 Buffer in quadruplicates for each strain and condition (three wells with sodium arsenite

988 (Sigma-Aldrich) and one well M9 as control). For tert-Butyl hydroperoxide (tBOOH)

989 stress assay, about 80 L4 C. elegans per condition were picked onto fresh RNAi

990 plates. Three days later, 20 day-three-old $C$. elegans were picked onto NGM plates

991 containing $15.4 \mathrm{mM}$ tBOOH (Sigma-Aldrich). The survival was scored every hour until

992 all animas died. Exploded animals were excluded from the statistics. The log-rank

993 (Mantel-Cox) method was used to test the null hypothesis and calculate $P$ values (JMP

994 software v.9.0.2.).

995

996 Oxidative stress assay by quantifying movement

997 C. elegans were collected from NGM plates and washed four times by centrifugation, 998 aspirating the supernatant and resuspending in fresh M9 buffer again. After the final 999 wash, the supernatant was removed, and $10 \mu \mathrm{l}$ of the $C$. elegans suspension pipetted 1000 into each well of a round-bottom 96-well microplate resulting in approximately 40 - 70 1001 animals per well. To prevent desiccation, the wells were filled up immediately with 1002 either $30 \mu \mathrm{l} 99$, or $30 \mu \mathrm{l}$ M9 containing $6.7 \mathrm{mM}$ or $18.7 \mathrm{mM}$ sodium arsenite yielding a 1003 final arsenite concentration of 0,5 , or $14 \mathrm{mM}$, respectively. Per $C$. elegans strain and 1004 conditions, we loaded two wells with M9 as control and six wells with either 5 or 14 $1005 \mathrm{mM}$ arsenite as technical replicates. The plate was closed, sealed with Parafilm and 1006 briefly stirred and then loaded into the wMicrotracker device (NemaMetrix). Data 1007 acquisition was performed for 50 hours, according to the manufacturer's instructions. 1008 The acquired movement dataset was analyzed using the dplyr 1009 (https://dplyr.tidyverse.org/reference/dplyr-package.html) and ggplot2 1010 (https://ggplot2.tidyverse.org) R packages. 


\section{$1012 \quad \mathbf{H}_{2} \mathbf{S}$ production capacity assay}

1013 The $\mathrm{H}_{2} \mathrm{~S}$ production capacity assay was adapted from Hine and colleagues ${ }^{33}$. $C$.

1014 elegans were harvested from NGM plates and washed four times by centrifugation

1015 and resuspension with M9 to remove residual bacteria. Approximately 3000 animals

1016 were collected as a pellet and mixed with the same volume of $2 x$ passive lysis buffer

1017 (Promega, E194A) on ice. Three freeze-thaw cycles were performed by freezing the

1018 samples in liquid nitrogen and thawing them again using a heat block set to $37^{\circ} \mathrm{C}$.

1019 Particles were removed by centrifuging at $12000 \mathrm{~g}$ for 10 minutes at $4^{\circ} \mathrm{C}$. The pellet

1020 was discarded, and the supernatant used further. The protein content of each sample

1021 was determined (BCA protein assay, Thermo scientific, 23225) and the sample sequentially diluted with distilled water to the required protein mass range, usually 25 - $200 \mu \mathrm{g}$ protein. To produce the lead acetate paper, we submerged chromatography

1024 paper (Whatman paper 3M (GE Healthcare, 3030-917)) in a 20 mM lead acetate (Lead

1025 (II) acetate trihydrate (Sigma, 215902-25G)) solution for one minute and then let it dry 1026 overnight. The fuel mix was prepared freshly by mixing Pyridoxal 5'-phosphate hydrate 1027 (Sigma, P9255-5G) and L-Cysteine (Sigma, C7352-25G) in Phosphate Buffered 1028 Saline on ice at final concentrations of $2.5 \mathrm{mM}$ and $25 \mathrm{mM}$, respectively. A 96-well 1029 plate was placed on ice, $80 \mu$ of each sample were loaded into each well and mixed 1030 with $20 \mu$ l fuel mix and subsequently covered using the lead acetate paper. The assay 1031 plate was then incubated at $37^{\circ} \mathrm{C}$ for 3 hours under a weight of approximately $1 \mathrm{~kg}$ to 1032 keep the lead acetate paper firmly in place. For analysis, the exposed lead acetate paper was imaged using a photo scanner. $\mathrm{H}_{2} \mathrm{~S}$ levels were quantified as the amount

1034 of lead sulfide captured on the paper, measured by the integrated density of each well 1035 area (Supplementary Table 12). Quantification of $\mathrm{H}_{2} \mathrm{~S}$ production was performed by 
measuring the integrated density using ImageJ, compared to a well next to it that contained no protein for background.

\section{Detection of $\mathrm{H}_{2} \mathrm{~S}$ levels by confocal microscopy}

1040 For the quantification of $\mathrm{H}_{2} \mathrm{~S}$ levels, worms were synchronized and grown at $20^{\circ} \mathrm{C}$ on 1041 regular NGM plates seeded with OP50-1 until they reached late L4 stage. At this point, 104250 animals per strain were transferred to fresh plates containing fluorescent $\mathrm{H}_{2} \mathrm{~S}$ probe

1043 to develop until the next day. Plates with $\mathrm{H}_{2} \mathrm{~S}$ sensor were made by spreading $100 \mu \mathrm{l}$ 1044 of $40 \mu \mathrm{M}$ MeRho-Az solution (in DMSO) ${ }^{34}$ on the plate surfaces and left to dry for at 1045 least $4 \mathrm{~h}$. On the control plates, the same volume of DMSO was spread as a vehicle 1046 control. On the day 1 of adulthood, worms were collected by picking, transferred to a 1047 tube containing M9 buffer and centrifuged for 1 minute at $400 \times \mathrm{g}$ to remove bacteria.

1048 Fixation was done in $2 \%$ PFA for 20 minutes at $37^{\circ} \mathrm{C}$ followed by incubation with $4 \%$ 1049 PFA for 20 minutes at RT with shaking. PFA was removed and worms were washed 10503 times with PBS supplemented with $0.01 \%$ Triton and twice with PBS. PBS was removed and mounting media (Ibidi, ref. 50001) was added directly to the tube. Worm suspension was transferred to the glass slide, covered with a cover slip and sealed with the nail polish. Samples were recorded on Leica TCS SP8 DLC Digital Light Sheet and Confocal microscope using 10X air objective and VIS (488 nm) laser. Obtained images were first processed with Worm-align open source pipeline for straightening and then analyzed for fluorescence intensity using Cell Profiler software.

\section{Persulfidation detection by confocal microscopy}

1059 Worms were synchronized by putting 15 gravid adults to lay eggs for $2 \mathrm{~h}$. Once the 1060 animals reached day 1 of adulthood, they were washed off the plates with M9 buffer, 
1061 collected into a $1.5 \mathrm{ml}$ tube, and centrifuged for 1 minute at $400 \times \mathrm{g}$. After 3 washes,

1062 M9 was removed, and worms were snap frozen in liquid nitrogen. Samples were

1063 defrosted by putting the tubes shortly in the water bath, and $200 \mu \mathrm{l}$ of $5 \mathrm{mM} \mathrm{NBF}-\mathrm{Cl}$ in

1064 PBS supplemented with $0.01 \%$ Triton was immediately added to tubes, followed by

1065 incubation at $37^{\circ} \mathrm{C}$ for $1 \mathrm{~h}$ with shaking. Worms were washed for 1 minute with ice-

1066 cold methanol while mixing, followed by 3 washes with PBS-Triton to remove excess

1067 NBF-Cl. Methanol/acetone fixation was performed on ice by incubating the samples

1068 in the ice-cold methanol for 5 minutes and then with the ice-cold acetone for 5 minutes.

1069 Acetone was removed, and 3 washes with PBS-Triton were performed. Samples were

1070 then again incubated with $5 \mathrm{mM} \mathrm{NBF-Cl}$ for 30 minutes at $37^{\circ} \mathrm{C}$ to ensure complete

1071 labelling. After the washes were performed in the same order as previously described,

1072 worms were incubated with $150 \mu$ of $25 \mu \mathrm{M}$ DAz-2:Cy-5 click mix for $1 \mathrm{~h}$ at $37^{\circ} \mathrm{C}$ with

1073 shaking. For the negative control, worms were incubated with $25 \mu \mathrm{M}$ DAz-2:Cy-5 click

1074 mix prepared without DAz-2. Samples were then washed 2 times for 5 minutes with

1075 ice-cold methanol and 3 times for 5 minutes with PBS-Triton to remove excess of the

1076 preclick product. DAPI staining was performed by incubating the samples with $300 \mathrm{nM}$

1077 DAPI solution in PBS-Triton for 5 minutes at RT with agitation. After several washes

1078 with PBS-Triton and PBS, worms were mounted on glass slide. Samples were

1079 recorded on Leica TCS SP8 DLC Digital Light Sheet and Confocal microscope using

1080 10X air objective and $405 \mathrm{~nm}$ laser for DAPI, $488 \mathrm{~nm}$ laser for NBF-adducts and 635

$1081 \mathrm{~nm}$ laser for PSSH. Obtained images were first processed with Worm-align open

1082 source pipeline for straightening and then analyzed for fluorescence intensity using

1083 Cell Profiler software.

1084

1085 Scoring of transgenic promoter-driven GFP 
1086 For Patf-4(uORF)::GFP, L4 stage transgenic animals were exposed to chemicals by

1087 top-coating with $500 \mu \mathrm{l}$ of each reagent (alpha-amanitin (Sigma \#A2263),

1088 cycloheximide (Sigma \#C7698), tunicamycin (Sigma \#T7765), sodium arsenite

1089 (Honeywell International \#35000)) or control (DMSO or M9 buffer) onto $6 \mathrm{~cm} \mathrm{NGM}$

1090 OP50 plates for 30 min to 4 hours, except that rapamycin (LC laboratories) was added

1091 to the NGM agar as described ${ }^{6}$. Then GFP fluorescent levels were either (1) scored

1092 or (2) quantified. (1) GFP scoring: Transgenic animals were first inspected with a

1093 dissecting scope while on still on the plate. GFP intensity was scored in the following

1094 categories: $0=$ none or very low GFP usually corresponding to untreated control, $1=$ 1095 low, 2= medium, and 3= high GFP fluorescence visible. Animals were washed off 1096 chemical treated plates, washed again at least twice, placed on OP50 NGM plates 1097 and were picked from there and mounted onto slides and GFP fluorescence was 1098 scored using a Zeiss AxioSKOP2 or a Tritech Research BX-51-F microscope with 1099 optimized triple-band filter-sets to distinguish autofluorescence from GFP at 40x as 1100 described $^{66}$. GFP was scored as the following: None: no GFP (excluding 1101 spermatheca), low: either only anterior or only posterior of the animal with weak GFP 1102 induction, Medium: both anterior and posterior of the animal with GFP but no GFP in 1103 the middle of the animal. High: GFP throughout the animal. $P$ values were determined 1104 by Chi ${ }^{2}$ test. (2) Quantification of GFP fluorescent levels: Animals were washed off 1105 reagent-containing plates, washed an additional two times, then placed into 24-well 1106 plates containing $0.06 \%$ tetramisole dissolved in M9 buffer to immobilize animals.

1107 Fluorescent pictures were taken with the same exposure settings (1s) at $10 x$ 1108 magnification using an Olympus Cellsens Standard Camera on an inverted 1109 microscope. GFP levels were assessed by drawing a line around the animal, 1110 measuring mean grey value and using the same area next to it for background using 
1111 ImageJ. The arbitrary fluorescent value corresponds to mean grey value of the animals

1112 minus the background.

1113

\section{Western blot}

1115 About 5000 C. elegans (L4 or day-1-adults indicated in figure legends) were sonicated

1116 in lysis buffer (RIPA buffer (ThermoFisher \#89900), 20 mM sodium fluoride (Sigma

1117 \#67414), $2 \mathrm{mM}$ sodium orthovanadate (Sigma \#450243), and protease inhibitor

1118 (Roche \#04693116001)) and kept on ice for 15 min before being centrifuged for 10

$1119 \min$ at $15^{\prime} 000 \times \mathrm{g}^{67}$. For equal loading, the protein concentration of the supernatant

1120 was determined with BioRad DC protein assay kit II (\#5000116) and standard curve

1121 with Albumin (Pierce \#23210). Samples were treated at $95^{\circ} \mathrm{C}$ for $5 \mathrm{~min}$, centrifuged for

$11221 \mathrm{~min}$ at $10^{\prime} 000 \times \mathrm{g}$ and $40 \mu \mathrm{g}$ protein was loaded onto NuPAGE Bis-Tris $10 \%$ Protein

1123 Gels (ThermoFisher \#NP0301BOX), and proteins were transferred to nitrocellulose

1124 membranes (Sigma \#GE10600002). Western blot analysis was performed under

1125 standard conditions with antibodies against Tubulin (1:500, Sigma \#T9026), GFP

1126 (1:1’000, Roche \#11814460001), Cystathionase/CTH (1:2000, abcam \#ab151769),

1127 Puromycin (1:10'000, Millipore \#MABE343), and Phospho-elF2 $\alpha$ (Ser51) (1:1'000,

1128 Cell Signaling \#9721). HRP-conjugated goat anti-mouse (1:2'000, Cell Signaling

1129 \#7076) and goat anti-rabbit (1:2'000, Cell Signaling \#7074) secondary antibodies were

1130 used to detect the proteins by enhanced chemiluminescence (Bio-Rad \#1705061). For

1131 loading control (i.e., Tubulin) either corresponding samples were run in parallel,

1132 membrane was cut if the size of Tubulin and protein of interest were not overlapping,

1133 membrane was incubated with loading control after detection of protein of interest on

1134 the same blot, or the blot was stripped (indicated in figure legends). For stripping,

1135 membranes were incubated for $5 \mathrm{~min}$ in acid buffer $(0.2 \mathrm{M}$ Glycin, $0.5 \mathrm{M} \mathrm{NaCl}$, pH set 
1136 to 2 with $\mathrm{HCl})$ and afterwards for 10 min in basic buffer $(0.5 \mathrm{M}$ Tris, $\mathrm{pH}$ set to 11 with

$1137 \mathrm{NaOH}$ ) and washed with TBS-T before blocking. Quantification of protein levels was

1138 determined by densitometry using ImageJ software and normalized to loading control

1139 (i.e., Tubulin). Uncropped blots are provided in the Supplementary Data File 1.

1140 
1141

1142

1143

1144

1145

1146

1147

1148

1149

1150

1151

1152

1153

1154

1155

1156

1157

1158

1159

1160

1161

1162

1163

1164

1165

1166

1167

1168

1169

1170

1171

1172

1173

1174

1175

1176

1177

1178

1179

1180

1181

1182

1183

1184

1185

1186

1187

1188

1189

\section{References}

1 Kenyon, C. J. The genetics of ageing. Nature 464, 504-512, doi:10.1038/nature08980 (2010).

2 Blackwell, T. K., Sewell, A. K., Wu, Z. \& Han, M. TOR Signaling in Caenorhabditis elegans Development, Metabolism, and Aging. Genetics 213, 329-360, doi:10.1534/genetics.119.302504 (2019).

3 Liu, G. Y. \& Sabatini, D. M. mTOR at the nexus of nutrition, growth, ageing and disease. Nat Rev Mol Cell Biol 21, 183-203, doi:10.1038/s41580-019-0199-y (2020).

4 Ben-Sahra, I. \& Manning, B. D. mTORC1 signaling and the metabolic control of cell growth. Curr Opin Cell Biol 45, 72-82, doi:10.1016/j.ceb.2017.02.012 (2017).

5 Bjedov, I. et al. Mechanisms of life span extension by rapamycin in the fruit fly Drosophila melanogaster. Cell Metab 11, 35-46, doi:10.1016/j.cmet.2009.11.010 (2010).

6 Robida-Stubbs, S. et al. TOR signaling and rapamycin influence longevity by regulating SKN-1/Nrf and DAF-16/FoxO. Cell Metab 15, 713-724, doi:10.1016/j.cmet.2012.04.007 (2012).

7 Pan, K. Z. et al. Inhibition of mRNA translation extends lifespan in Caenorhabditis elegans. Aging Cell 6, 111-119, doi:10.1111/j.1474-9726.2006.00266.x (2007).

8 Curran, S. P. \& Ruvkun, G. Lifespan regulation by evolutionarily conserved genes essential for viability. PLoS Genet 3, e56, doi:10.1371/journal.pgen.0030056 (2007).

9 Syntichaki, P., Troulinaki, K. \& Tavernarakis, N. eIF4E function in somatic cells modulates ageing in Caenorhabditis elegans. Nature 445, 922-926, doi:10.1038/nature05603 (2007).

10 Wang, J. et al. RNAi screening implicates a SKN-1-dependent transcriptional response in stress resistance and longevity deriving from translation inhibition. PLoS Genet 6, doi:10.1371/journal.pgen.1001048 (2010).

11 Howard, A. C., Rollins, J., Snow, S., Castor, S. \& Rogers, A. N. Reducing translation through eIF4G/IFG-1 improves survival under ER stress that depends on heat shock factor HSF-1 in Caenorhabditis elegans. Aging Cell 15, 1027-1038, doi:10.1111/acel.12516 (2016).

12 Hansen, M. et al. Lifespan extension by conditions that inhibit translation in Caenorhabditis elegans. Aging Cell 6, 95-110, doi:10.1111/j.1474-9726.2006.00267.x (2007).

13 Harding, H. P. et al. An integrated stress response regulates amino acid metabolism and resistance to oxidative stress. Mol Cell 11, 619-633, doi:10.1016/s10972765(03)00105-9 (2003).

14 Costa-Mattioli, M. \& Walter, P. The integrated stress response: From mechanism to disease. Science 368, doi:10.1126/science.aat5314 (2020).

15 Derisbourg, M. J., Wester, L. E., Baddi, R. \& Denzel, M. S. Mutagenesis screen uncovers lifespan extension through integrated stress response inhibition without reduced mRNA translation. Nat Commun 12, 1678, doi:10.1038/s41467-021-21743-x (2021).

16 Sidrauski, C. et al. Pharmacological brake-release of mRNA translation enhances cognitive memory. Elife 2, e00498, doi:10.7554/eLife.00498 (2013).

17 Mittal, N. et al. The Gcn4 transcription factor reduces protein synthesis capacity and extends yeast lifespan. Nat Commun 8, 457, doi:10.1038/s41467-017-00539-y (2017).

$18 \mathrm{Hu}, \mathrm{Z}$. et al. Ssd1 and Gcn2 suppress global translation efficiency in replicatively aged yeast while their activation extends lifespan. Elife 7, doi:10.7554/eLife.35551 (2018). 
119019 Horn, M. et al. Hexosamine Pathway Activation Improves Protein Homeostasis through the Integrated Stress Response. iScience 23, 100887, doi:10.1016/j.isci.2020.100887 (2020).

20 Park, Y., Reyna-Neyra, A., Philippe, L. \& Thoreen, C. C. mTORC1 Balances Cellular Amino Acid Supply with Demand for Protein Synthesis through Post-transcriptional Control of ATF4. Cell Rep 19, 1083-1090, doi:10.1016/j.celrep.2017.04.042 (2017).

21 Rousakis, A. et al. The general control nonderepressible-2 kinase mediates stress response and longevity induced by target of rapamycin inactivation in Caenorhabditis elegans. Aging Cell 12, 742-751, doi:10.1111/acel.12101 (2013).

22 Pakos-Zebrucka, K. et al. The integrated stress response. EMBO Rep 17, 1374-1395, doi:10.15252/embr.201642195 (2016).

23 Kulalert, W., Sadeeshkumar, H., Zhang, Y. K., Schroeder, F. C. \& Kim, D. H. Molecular Determinants of the Regulation of Development and Metabolism by Neuronal eIF2alpha Phosphorylation in Caenorhabditis elegans. Genetics 206, 251263, doi:10.1534/genetics.117.200568 (2017).

$24 \mathrm{Li}$, W. J. et al. Insulin signaling regulates longevity through protein phosphorylation in Caenorhabditis elegans. Nat Commun 12, 4568, doi:10.1038/s41467-021-24816-Z (2021).

25 Blackwell, T. K., Steinbaugh, M. J., Hourihan, J. M., Ewald, C. Y. \& Isik, M. SKN1/Nrf, stress responses, and aging in Caenorhabditis elegans. Free Radic Biol Med 88, 290-301, doi:10.1016/j.freeradbiomed.2015.06.008 (2015).

26 Ewald, C. Y., Landis, J. N., Porter Abate, J., Murphy, C. T. \& Blackwell, T. K. Dauer-independent insulin/IGF-1-signalling implicates collagen remodelling in longevity. Nature 519, 97-101, doi:10.1038/nature14021 (2015).

27 Quiros, P. M. et al. Multi-omics analysis identifies ATF4 as a key regulator of the mitochondrial stress response in mammals. J Cell Biol 216, 2027-2045, doi:10.1083/jcb.201702058 (2017).

28 Han, J. et al. ER-stress-induced transcriptional regulation increases protein synthesis leading to cell death. Nat Cell Biol 15, 481-490, doi:10.1038/ncb2738 (2013).

29 Hoshino, A. et al. The ADP/ATP translocase drives mitophagy independent of nucleotide exchange. Nature 575, 375-379, doi:10.1038/s41586-019-1667-4 (2019).

30 Zhu, J. et al. Transsulfuration Activity Can Support Cell Growth upon Extracellular Cysteine Limitation. Cell Metab 30, 865-876 e865, doi:10.1016/j.cmet.2019.09.009 (2019).

31 Miller, R. A. et al. Methionine-deficient diet extends mouse lifespan, slows immune and lens aging, alters glucose, T4, IGF-I and insulin levels, and increases hepatocyte MIF levels and stress resistance. Aging Cell 4, 119-125, doi:10.1111/j.14749726.2005.00152.x (2005).

32 Miller, D. L. \& Roth, M. B. Hydrogen sulfide increases thermotolerance and lifespan in Caenorhabditis elegans. Proc Natl Acad Sci U S A 104, 20618-20622, doi:10.1073/pnas.0710191104 (2007).

33 Hine, C. et al. Endogenous hydrogen sulfide production is essential for dietary restriction benefits. Cell 160, 132-144, doi:10.1016/j.cell.2014.11.048 (2015).

34 Hammers, M. D. et al. A Bright Fluorescent Probe for H2S Enables AnalyteResponsive, 3D Imaging in Live Zebrafish Using Light Sheet Fluorescence Microscopy. J Am Chem Soc 137, 10216-10223, doi:10.1021/jacs.5b04196 (2015).

35 Zivanovic, J. et al. Selective Persulfide Detection Reveals Evolutionarily Conserved Antiaging Effects of S-Sulfhydration. Cell Metab 30, 1152-1170 e1113, doi:10.1016/j.cmet.2019.10.007 (2019). 
36 Paul, B. D. \& Snyder, S. H. H2S: A Novel Gasotransmitter that Signals by Sulfhydration. Trends Biochem Sci 40, 687-700, doi:10.1016/j.tibs.2015.08.007 (2015).

37 Meng, J. et al. Global profiling of distinct cysteine redox forms reveals wide-ranging redox regulation in C. elegans. Nat Commun 12, 1415, doi:10.1038/s41467-02121686-3 (2021).

38 Molenaars, M. et al. A Conserved Mito-Cytosolic Translational Balance Links Two Longevity Pathways. Cell Metab 31, 549-563 e547, doi:10.1016/j.cmet.2020.01.011 (2020).

39 Wei, Y. \& Kenyon, C. Roles for ROS and hydrogen sulfide in the longevity response to germline loss in Caenorhabditis elegans. Proc Natl Acad Sci U S A 113, E28322841, doi:10.1073/pnas.1524727113 (2016).

40 Zinzalla, V., Stracka, D., Oppliger, W. \& Hall, M. N. Activation of mTORC2 by association with the ribosome. Cell 144, 757-768, doi:10.1016/j.cell.2011.02.014 (2011).

41 Sarbassov, D. D. et al. Prolonged rapamycin treatment inhibits mTORC2 assembly and Akt/PKB. Mol Cell 22, 159-168, doi:10.1016/j.molcel.2006.03.029 (2006).

42 Lamming, D. W. et al. Rapamycin-induced insulin resistance is mediated by mTORC2 loss and uncoupled from longevity. Science 335, 1638-1643, doi:10.1126/science.1215135 (2012).

43 Rogers, A. N. et al. Life span extension via eIF4G inhibition is mediated by posttranscriptional remodeling of stress response gene expression in C. elegans. Cell Metab 14, 55-66, doi:10.1016/j.cmet.2011.05.010 (2011).

$44 \mathrm{Li}, \mathrm{W}$., Li, X. \& Miller, R. A. ATF4 activity: a common feature shared by many kinds of slow-aging mice. Aging Cell 13, 1012-1018, doi:10.1111/acel.12264 (2014).

45 Li, W. \& Miller, R. A. Elevated ATF4 function in fibroblasts and liver of slow-aging mutant mice. J Gerontol A Biol Sci Med Sci 70, 263-272, doi:10.1093/gerona/glu040 (2015).

46 Hine, C., Zhu, Y., Hollenberg, A. N. \& Mitchell, J. R. Dietary and Endocrine Regulation of Endogenous Hydrogen Sulfide Production: Implications for Longevity. Antioxid Redox Signal 28, 1483-1502, doi:10.1089/ars.2017.7434 (2018).

47 Akaike, T. et al. Cysteinyl-tRNA synthetase governs cysteine polysulfidation and mitochondrial bioenergetics. Nat Commun 8, 1177, doi:10.1038/s41467-017-01311-y (2017).

48 Valvezan, A. J. \& Manning, B. D. Molecular logic of mTORC1 signalling as a metabolic rheostat. Nat Metab 1, 321-333, doi:10.1038/s42255-019-0038-7 (2019).

49 Heintz, C. et al. Splicing factor 1 modulates dietary restriction and TORC1 pathway longevity in C. elegans. Nature 541, 102-106, doi:10.1038/nature20789 (2017).

50 Lee, G. et al. Post-transcriptional Regulation of De Novo Lipogenesis by mTORC1S6K1-SRPK2 Signaling. Cell 171, 1545-1558 e1518, doi:10.1016/j.cell.2017.10.037 (2017).

51 Paul, B. D. \& Snyder, S. H. H(2)S signalling through protein sulfhydration and beyond. Nat Rev Mol Cell Biol 13, 499-507, doi:10.1038/nrm3391 (2012).

52 Filipovic, M. R., Zivanovic, J., Alvarez, B. \& Banerjee, R. Chemical Biology of H2S Signaling through Persulfidation. Chem Rev 118, 1253-1337, doi:10.1021/acs.chemrev.7b00205 (2018).

53 Longchamp, A. et al. Amino Acid Restriction Triggers Angiogenesis via GCN2/ATF4 Regulation of VEGF and H2S Production. Cell 173, 117-129 e114, doi:10.1016/j.cell.2018.03.001 (2018). 
54 Islam, K. N., Polhemus, D. J., Donnarumma, E., Brewster, L. P. \& Lefer, D. J. Hydrogen Sulfide Levels and Nuclear Factor-Erythroid 2-Related Factor 2 (NRF2) Activity Are Attenuated in the Setting of Critical Limb Ischemia (CLI). $J$ Am Heart Assoc 4, doi:10.1161/JAHA.115.001986 (2015).

55 Stadler, M., Artiles, K., Pak, J. \& Fire, A. Contributions of mRNA abundance, ribosome loading, and post- or peri-translational effects to temporal repression of C. elegans heterochronic miRNA targets. Genome Res 22, 2418-2426, doi:10.1101/gr.136515.111 (2012).

56 Fiorese, C. J. et al. The Transcription Factor ATF5 Mediates a Mammalian Mitochondrial UPR. Curr Biol 26, 2037-2043, doi:10.1016/j.cub.2016.06.002 (2016).

57 Venz, R., Korosteleva, A., Jongsma, E. \& Ewald, C. Y. Combining Auxin-Induced Degradation and RNAi Screening Identifies Novel Genes Involved in Lipid Bilayer Stress Sensing in Caenorhabditis elegans. G3 (Bethesda) 10, 3921-3928, doi:10.1534/g3.120.401635 (2020).

58 Ewald, C. Y. et al. NADPH oxidase-mediated redox signaling promotes oxidative stress resistance and longevity through memo-1 in C. elegans. Elife 6, doi:10.7554/eLife.19493 (2017).

59 Schmidt, E. K., Clavarino, G., Ceppi, M. \& Pierre, P. SUnSET, a nonradioactive method to monitor protein synthesis. Nat Methods 6, 275-277, doi:10.1038/nmeth.1314 (2009).

60 Steinbaugh, M. J. et al. Lipid-mediated regulation of SKN-1/Nrf in response to germ cell absence. Elife 4, doi:10.7554/eLife.07836 (2015).

61 Kim, W., Underwood, R. S., Greenwald, I. \& Shaye, D. D. OrthoList 2: A New Comparative Genomic Analysis of Human and Caenorhabditis elegans Genes. Genetics 210, 445-461, doi:10.1534/genetics.118.301307 (2018).

62 Patro, R., Duggal, G., Love, M. I., Irizarry, R. A. \& Kingsford, C. Salmon provides fast and bias-aware quantification of transcript expression. Nat Methods 14, 417-419, doi:10.1038/nmeth.4197 (2017).

63 Stroustrup, N. et al. The Caenorhabditis elegans Lifespan Machine. Nat Methods 10, 665-670, doi:10.1038/nmeth.2475 (2013).

64 Gentleman, R. I. a. R. R: A Language for Data Analysis and Graphics. Journal of Computational and Graphical Statistics 5, 299-314 (1996).

65 Ewald, C. Y., Hourihan, J. M. \& Blackwell, T. K. Oxidative Stress Assays (arsenite and tBHP) in Caenorhabditis elegans. Bio Protoc 7, doi:10.21769/BioProtoc. 2365 (2017).

66 Teuscher, A. C. \& Ewald, C. Y. Overcoming Autofluorescence to Assess GFP Expression During Normal Physiology and Aging in Caenorhabditis elegans. Bio Protoc 8, doi:10.21769/BioProtoc.2940 (2018).

67 Hourihan, J. M., Moronetti Mazzeo, L. E., Fernandez-Cardenas, L. P. \& Blackwell, T. K. Cysteine Sulfenylation Directs IRE-1 to Activate the SKN-1/Nrf2 Antioxidant Response. Mol Cell 63, 553-566, doi:10.1016/j.molcel.2016.07.019 (2016). 